\title{
REDUCIBILITY OF DIFFERENTIAL EQUATIONS AND PSEUDO-ISOMORPHISM OF AUTOMORPHISM PSEUDO-GROUPS
}

\author{
KAZUSHIGE UENO
}

\section{Introduction}

Lie's theory of the integration for a category $C$ of differential equations is composed of the following four kinds of problems:

(1) Determine all canonical forms of differential equations belonging to $C$. e.

(2) Discriminate the canonical form of a differential equation belonging to

(3) Translate a differential equation in $\mathcal{C}$ to its canonical form.

(4) Integrate all canonical forms in $C$.

Problem (1) is a classification problem, and Problem (2) is an equivalence problem.

For example, let $\mathcal{C}(M)$ be the set of local vector fields with no singularity on a manifold $M$. Then it is well-known that each element $X$ of $C(M)$ possesses the caonical form $\partial / \partial x$ as a germ. That is, in this case, Problems (1), (2) and (4) are trivial. Problem (3) is to seek for a local transformation of $X$ to $\partial / \partial x$.

Now we shall pose the following problem: Let $\mathcal{G}$ be a pseudo-group on a manifold $Q$, and let $\mathcal{C}_{\Gamma}$ be the set of a differential equation $E$ such that the automorphism pseudo-group $\mathscr{Q}(E)$ of $E$ is equal to $\Gamma$. Then the problem to consider is to classify the category $\bigodot_{\Gamma}$.

As the associated problem to this, we consider the reducibility of a differential equation $E$ to another differential equation $E^{\prime}$.

This is similar to the reduction of the classification problem of pseudogroups to the primitive case.

Let $\left(Q, Q^{\prime}, \pi\right)$ be a fibred manifold, and let $E$ or $E^{\prime}$ be a differential

Received February 7, 1977. 
equation at $j_{x}^{\alpha}(f) \in J^{\alpha}(N, Q)$ or $j_{x}^{\alpha^{\prime}}\left(f^{\prime}\right) \in J^{\alpha^{\prime}}\left(N, Q^{\prime}\right)$, respectively. Roughly speaking, the pair $\left(E, E^{\prime}\right)$ is called a reduced pair if $\pi$ induces a one to one correspondence between the solution space of $E$ and that of $E^{\prime}$.

The purpose of this paper is to formulate such a reduced pair of differential equations and to characterize it by their automorphism pseudo-groups and their resolvent systems.

In $\S 1$, we shall introduce the notion of orbit systems of a weak Lie algebra sheaf.

In $\S 2$, we shall state the structure of a differential equation $E$ as a family of orbit systems of the weak Lie algebra sheaf which is induced from the automorphism pseudo-group $Q(E)$ of $E$. The family is parametrized by the solution space of a resolvent differential equation of $E$ (Corollary 2.2.1). E. Vessiot or H. H. Johnson refers to the contents of this section in [7] or [1].

In §3, we shall reduce the equivalence problem of differential equations to that of their resolvent differential equations (Proposition 3.3.1). This will be applied to the compatibility of reduction and equivalence in $\$ 6$.

In $\S 4$, we shall define the reducibility of a differential equation $E$ and prove that under some conditions the reduction is of orbit-system-preserving (Lemma 4.1.1). As one of the applications of Lemma 4.1.1, we shall show that if $E$ is an $\mathfrak{L}_{\mathscr{Q}(E)}$-orbit system, any reduced form $E^{\prime}$ is also an $\mathfrak{L}_{\mathscr{Q}(E)}$-orbit system. Furthermore we shall prove that if $E$ is $\mathcal{Q}(E)$-automorphic, then $E^{\prime}$ is $\mathbb{Q}\left(E^{\prime}\right)$-automorphic (Proposition 4.1.1).

In $\S 5$, we shall state the notion of pseudo-isomorphism of pseudo-groups (Definition 5.1.1) which is given in [3] in a vague form, and shall characterize the reducibility of a differential equation $E$ to a differential equation $E^{\prime}$ by using the pseudo-isomorphism of $\mathbb{Q}(E)$ to $\mathscr{Q}\left(E^{\prime}\right)$ (Theorems 5.2.1, 5.2.2).

In $\$ 6$, we shall study the compatibility of reduction and equivalence of differential equations, namely, for two pairs $\left(E_{1}, E_{1}^{\prime}\right)$ and $\left(E_{2}, E_{2}^{\prime}\right)$ satisfying some conditions, if $E_{1}$ is isomorphic to $E_{2}$, then $E_{1}^{\prime}$ is isomorphic to $E_{2}^{\prime}$ in a sense (Theorem 6.2.1).

In $\$ 7$, we shall give an example of a pseudo-group which is $k$-closed at $(x, f)$ for some integer $k$ where $f$ is a local immersion (Proposition 7.1.1).

The completeness and the order of pseudo-groups are explained in $\$ 8$ and $\$ 9$ as appendices.

Throughout this paper, we assume the differentiability of class $C^{\omega}$. By a differential equation at $p \in J^{l}(N, Q)$, we mean a system of functions defined on a neighborhood of $p$. For a pseudo-group $\Gamma$, we always assume that any element of $\Gamma$ is near to the identity. As to a (weak) Lie algebra sheaf, refer to [5] or [6]. 


\section{Index of notation}

$I(E)$ : the set of integral points of a differential equation $E \ldots \ldots \ldots \ldots \ldots \ldots . . . . . . . . . .4$

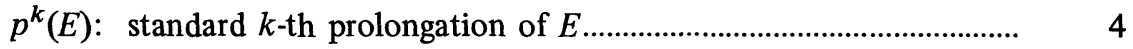

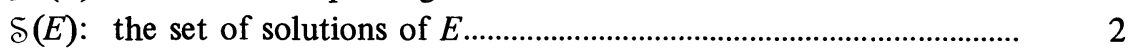

$\mathcal{Q}(E)$ : automorphism pseudo-group of $E$................................................ 2

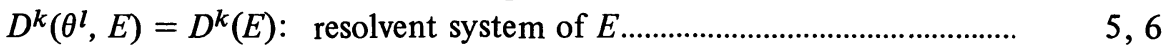

ขै ${ }^{k}\left(\theta^{l}, E\right):$ resolvent space of $E$............................................................ 4

$D_{E}^{k}$ : involutive distribution induced from a differential equation $E$......... 6

$\Re(\Gamma)$ : normalizer of a pseudo-group $\Gamma$ on $Q$ in the pseudo-group of all

local transformations of $Q$................................................................ 5

$D\left(\theta^{l}, \Re(\Gamma)\right)=D(\Re(\Gamma))$ : pseudo-group induced from $\Re(\Gamma)$ via the map

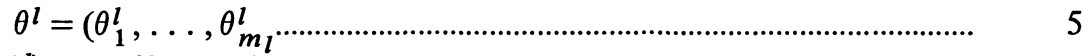

$F\left(\theta^{l}\right): \quad \Gamma$-differential invariant ..................................................................... 3

$\mathcal{L}:$ a sheaf of vector fields associated to a pseudo-group $\Gamma$..................... 3

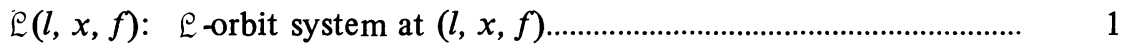

\section{Orbit systems}

1.1. We denote by $J^{l}(N, Q)$ the space of $l$-jets $j_{x}^{l}(f)$ of local maps $f$ of a manifold $N$ to another manifold $Q$, and if $\operatorname{dim} N \geqslant \operatorname{dim} Q \geqslant 1$, we denote by $\tilde{J}^{l}(N, Q)$ the open submanifold of $J^{l}(N, Q)$ which is the $l$-jet space of local submersions. If $\operatorname{dim} Q>\operatorname{dim} N \geqslant 1, \tilde{J}^{l}(N, Q)$ means the $l$-jet space of local immersions which is also an open submanifold of $J^{l}(N, Q)$. In case $l=0, J^{0}(N, Q)$ or $\tilde{J}^{0}(N, Q)$ means the manifold $N \times Q$, and $j_{x}^{0}(f)$ the point $(x, f(x)) \in N \times Q$.

Let $\phi$ be any local diffeomorphism of $Q$. Then $\phi$ is prolonged to a local diffeomorphism $\phi^{(l)}$ of $\tilde{J}^{l}(N, Q)$, which is defined by $\phi^{(l)}\left(j_{x}^{l}(f)\right)=j_{x}^{l}(\phi \circ f)$, $j_{x}^{l}(f) \in \tilde{J}^{l}(N, Q)$.

Let $X$ be a germ of a local vector field on $Q$. Then, by considering the local 1-parameter group of local transformations generated by $X$, we can easily see that $X$ is prolonged to a germ $X^{(l)}$ of a local vector field on $\tilde{J}^{l}(N, Q)$. Therefore a weak Lie algebra sheaf $\mathcal{L}$ on $Q$ is prolonged to a weak Lie algebra sheaf $\mathcal{L}^{(l)}$ on $\tilde{J}^{l}(N, Q)$.

Let ${ }^{0} \mathcal{L}_{p}^{(l)}$ denote the isotropy of the stalk $\mathcal{L}_{p}^{(l)}$, and set $D_{p}^{(l)}=\mathcal{L}_{p}^{(l) / 0} \mathcal{L}_{p}^{(l)}$.

Definition 1.1.1. A weak Lie algebra sheaf $\mathcal{L}$ on $Q$ is said to be $(l, N)$-regular if $\operatorname{dim} D_{p}^{(l)}$ is constant. If $\mathcal{E}$ is $(l, N)$-regular for any integer $l \geqslant 0$, then $\mathcal{L}$ is said to be $N$-regular. 
Definition 1.1.2. A function $\phi$ given on an open subset $\theta^{l}$ of $\tilde{J}^{l}(N, Q)$ is called a differential invariant of $\mathcal{L}$ at $j_{x}^{l}(f) \in \mathcal{O}^{l}$ if there is a neighborhood $\mathcal{Q}^{l} \subset \mathcal{O}^{l}$ of $j_{x}^{l}(f)$ such that, for any $p \in \mathcal{Q}^{l}$ and any germ $X^{(l)}$ of $\mathfrak{L}_{p}^{(l)}$, we have $X^{(l)} \cdot \phi_{p}=0$ where $\phi_{p}$ is a germ of $\phi$ at $p$.

Note that if $\mathcal{L}$ is $N$-regular, then a differential invariant of $\mathcal{L}$ at $j_{x}^{l}(f)$ is a first integral of the involutive distribution $D^{(l)}$ at $j_{x}^{l}(f)$.

Definition 1.1.3. A fundamental system of first integrals of $D^{(l)}$ at $j_{x}^{l}(f)$ is called a fundamental system of differential invariants of $\mathcal{L}$ at $j_{x}^{l}(f)$.

Suppose $\mathcal{E}$ is an $N$-regular weak Lie algebra sheaf on $Q$.

Lemma 1.1.1. Let $\left\{\theta_{j}^{l}\right\}_{j=1}^{m_{l}}$ and $\left\{\tilde{\theta}_{j}^{l}\right\}_{j=1}^{m_{l}}$ be two fundamental system of differential invariants of $\mathcal{L}$ at $j_{x_{0}}^{l}(f)$, and set $\lambda_{j}(x)=\theta_{j}^{l}\left(j_{x}^{l}(f)\right)$ and $\tilde{\lambda}_{j}(x)=$ $\tilde{\theta}_{j}^{l}\left(j_{x}^{l}(f)\right)$. We denote by $(*)^{l}$ (respectively $\left.(\tilde{*})^{l}\right)$ the system of differential equations generated by $\theta_{j}^{l}-\lambda_{j}\left(1 \leqslant j \leqslant m_{l}\right)$ (respectively $\tilde{\theta}_{j}^{l}-\tilde{\lambda}_{j}\left(1 \leqslant j \leqslant m_{l}\right)$ ). Then the set of solutions of $(*)^{l}$ is equal to that of $(\tilde{*})^{l}$.

Proof. We have the analytic expressions $\tilde{\theta}_{j}^{l}=\xi_{j}\left(\theta_{1}^{l}, \cdots, \theta_{m}^{l}\right), 1 \leqslant j \leqslant m_{l}$. Then we get $\tilde{\lambda}_{j}=\xi_{j}\left(\lambda_{1}, \cdots, \lambda_{m_{l}}\right), 1 \leqslant j \leqslant m_{l}$. Let $s$ be any solution of $(*)^{l}$. Then $\boldsymbol{\theta}_{j}^{l}\left(j_{x}^{l}(s)\right)=\lambda_{j}(x), \quad 1 \leqslant j \leqslant m_{l}$. Therefore we have $\tilde{\theta}_{j}\left(j_{x}^{l}(s)\right)=$ $\xi_{j}\left(\theta_{1}^{l}\left(j_{x}^{l}(s)\right)\right), \cdots, \theta_{m_{l}}^{l}\left(j_{x}^{l}(s)\right)=\xi_{j}\left(\lambda_{1}(x), \cdots, \lambda_{m_{l}}(x)\right)=\tilde{\lambda}_{j}(x), \quad 1 \leqslant j<m_{l}$. This implies that $s$ is a solution of $(\tilde{*})^{l}$. Similarly any solution of $(\tilde{*})^{l}$ is also a solution of $(*)^{l}$. Hence the proof is completed.

Definition 1.1.4. The differential equation $(*)^{l}$ is called the $\mathcal{L}$-orbit system at $\left(l, x_{0}, f\right)$ and denoted by $\mathcal{L}\left(l, x_{0}, f\right)$.

\section{Structures of differential equations}

2.1. Let $E$ be a differential equation at $j_{x}^{l}(f) \in \tilde{J}^{l}(N, Q)$. We denote by $\delta(E)$ the set of solutions of $E$. For any neighborhood $\mathcal{Q}^{k}$ of $j_{x}^{k}(f)$, we denote by $\delta(E) \mid Q^{k}$ the set of solutions $s$ of $E$ such that the image of $j^{k}(s) \subset \mathcal{Q}^{k}$.

Definition 2.1.1. A differential equation $E^{1}$ at $j_{x}^{l}\left(f^{1}\right)$ is said to be isomorphic to a differential equation $E^{2}$ at $j_{x}^{l}\left(f^{2}\right)$ if there exist a diffeomorphism $\psi$ of a neighborhood $\mathcal{Q}^{1}$ of $f^{1}(x) \in Q$ to a neighborhood $\mathcal{Q}^{2}$ of $f^{2}(x) \in Q$ and a neighborhood $\mathscr{W}$ of $x \in N$ such that $\delta\left(E^{1}\right) \mid \mho \times \mathcal{Q}^{1} \ni s$ if and only if $\psi \circ s \in \delta\left(E^{2}\right) \mid \mho \times \mathcal{Q}^{2} . \psi$ is called an isomorphism of $E^{1}$ to $E^{2}$.

Definition 2.1.2. If $E^{1}=E^{2}=E$ and $f^{1}=f^{2}=f$, we denote by $\mathcal{Q}(E)$ the pseudo-group on a neighborhood of $f(x)$ generated by the set of isomorphisms of $E$ to $E . \mathbb{Q}(E)$ is called the automorphism pseudo-group of $E$, and each element of $\mathcal{Q}(E)$ is called an automorphism of $E$.

2.2. Let $\Gamma$ be a pseudo-group on $Q$ such that $\varrho_{\Gamma}$ is an $N$-regular weak Lie algebra sheaf, where $\mathscr{E}_{T}$ is the sheaf of germs of local vector fields $X$ such that 
the local 1-parameter group of local transormations generated by $X$ is contained in $\Gamma$. Let $\left\{\theta_{j}^{l}\right\}_{j=1}^{m_{l}}$ be a fundamental system of differential invariants of $\mathscr{L}_{T}$ at $j_{x_{0}}^{l}(f)$. We set $\theta^{l}=\left(\theta_{1}^{l}, \cdots, \theta_{m}^{l}\right)$. Then $\theta^{l}$ is a submersion of a neighborhood $\mathcal{Q}^{l}$ of $j_{x_{0}}^{l}(f)$ onto a neighborhood $W$ of $\theta^{l}\left(j_{x_{0}}^{l}(f)\right)=$ $\left(\theta_{1}^{l}\left(j_{x_{0}}^{l}(f)\right), \cdots, \theta_{m_{l}}^{l}\left(j_{x_{0}}^{l}(f)\right)\right) \in \mathbf{R}^{m_{l}}$. Let $\rho_{l}^{l+k}$ be the projection of $\tilde{J}^{l+k}(N, Q)$ onto $\tilde{J}^{l}(N, Q)$ and set $Q^{l+k}=\left(\rho_{l}^{l+k}\right)^{-1}\left(\mathcal{Q}^{l}\right)$. Similarly let $\alpha^{l}$ and $\beta^{l}$ be the projection of $J^{l}(N, Q)$ onto $N$ and $Q$ respectively. We denote by $p^{k} \theta^{l}$ the map of $\mathcal{Q}^{l+k}$ to $J^{k}(\mathcal{V}, W) \subset J^{k}\left(N, \mathbf{R}^{m_{l}}\right)$, where $\mathcal{V}=\alpha^{l}\left(\mathcal{Q}^{l}\right)$, defined by $p^{k} \theta^{l}$ $\left(j_{x}^{l+k}(f)\right)=j_{x}^{k}\left(\theta^{l}\left(j^{l}(f)\right)\right)$. We set $q^{k}=j_{x_{0}}^{k}\left(\theta^{l}\left(j^{l}(f)\right)\right)$. Then $q^{k} \in J^{k}(\mathcal{V}, W)$. For any function $F$ locally defined at $q^{k}$, we set $F\left(\theta^{l}\right)=\left(p^{k} \theta^{l}\right)^{*} F$, which is a function locally defined at $J_{x_{0}}^{l+k}(f)$. Let $\left\{\tilde{\theta}_{j}^{l}\right\}_{j=1}^{m_{l}}$ be any other fundamental system of differential invariants of $E_{\Gamma}$ at $j_{x_{0}}^{l}(f)$. If $y$ is a differential invariant of $\mathcal{E}_{\Gamma}$ at $j_{x_{0}}^{l+k}(f)$ of the form $F\left(\theta^{l}\right)$, then $y$ is also of the form $\tilde{F}\left(\tilde{\theta}^{l}\right)$.

Definition 2.2.1. A differential invariant of $\varrho_{\Gamma}$ at $j_{x_{0}}^{l+k}(f)$ of the form $F\left(\theta^{l}\right)$ is called a $\Gamma$-differential invariant of type $l$ at $j_{x_{0}}^{l+k}(f)$.

Definition 2.2.2. A family of linearly independent differential invariants $\mathcal{K}=\left\{\mathfrak{h}_{j}\right\}_{j=1}^{r}$ of $\mathfrak{E}_{\Gamma}$ at $j_{x_{0}}^{l+k}(f)$ is called a $\Gamma$-family at $j_{x_{0}}^{l+k}(f)$ of type $(l, r)$ if $\mathcal{H}$ satisfies the following conditions:

(1) $\mathfrak{h}_{j}$ is a $\Gamma$-differential invariant of type $l$ at $j_{x_{0}}^{l+k}(f), 1 \leqslant j \leqslant r$.

(2) The differential equation at $j_{x_{0}}^{l+k}(f)$ generated by $\mathfrak{h}_{j}, 1 \leqslant j \leqslant r$, possesses a solution.

(3) The automorphism pseudo-group of the differential equation is equal to $\Gamma$ on a neighborhood of $f\left(x_{0}\right)$.

Proposition 2.2.1. Suppose $\mathcal{H}=\left\{\mathfrak{h}_{j}\right\}_{j=1}^{r}$ is a $\Gamma$-family at $j_{x_{0}}^{l+k}(f)$ of type $(l, r)$. Then $\Gamma$ is locally determined at $f\left(x_{0}\right)$ by $\mathcal{H}$. Namely, assume that each $\mathfrak{h}_{j}$ is defined on a neighborhood $V^{l+k}$ of $j_{x_{0}}^{l+k}(f)$. We denote by $\tilde{\Gamma}$ a pseudo-group on $\beta^{l+k}\left(V^{l+k}\right)$ which is defined by the following way $\tilde{\Gamma} \ni \phi: \mathcal{U} \rightarrow \mathcal{V}$ if and only if $\phi^{(l+k)^{*}} \mathfrak{h}_{j}=\mathfrak{h}_{j}(1 \leqslant j \leqslant r)$ on $V^{l+k} \cap \tilde{J}^{l+k}(N$, Q $)$. Then we have $\tilde{\Gamma}=\Gamma$ on a neighborhood थิ $\subset \beta^{l+k}\left(V^{l+k}\right)$ of $f\left(x_{0}\right)$.

Proof. Since $\mathfrak{h}_{j}$ is a differential invariant of $\varrho_{\Gamma}$ at $j_{x_{0}}^{l+k}(f)$, it is clear that $\tilde{\Gamma}$ contains $\Gamma$ on a neighborhood of $f\left(x_{0}\right)$. On the other hand, $\tilde{\Gamma}$ is contained in the automorphism pseudo-group of the differential equation generated by $\mathfrak{h}_{j}$ $(1 \leqslant j \leqslant r)$, which is, by the assumption that $\mathcal{K}$ is a $\Gamma$-family at $j_{x_{0}}^{l+k}(f)$ of type $(l, r)$, equal to $\Gamma$ on a neighborhood of $f\left(x_{0}\right)$. Therefore $\tilde{\Gamma}$ is equal to $\Gamma$ on a neighborhood थี $\subset \beta^{l+k}\left(V^{l+k}\right)$ of $f\left(x_{0}\right)$.

2.3. Definition 2.3.1. A family $\Theta^{l}=\left\{\theta_{j}^{l}\right\}_{j=1}^{m}$ of such functions that $\theta_{j}^{l}$ $(1 \leqslant j \leqslant m)$ are defined on a neighborhood $\tilde{Q}^{l}$ of $j_{x_{0}}^{l}(f) \in \tilde{J}^{l}(N, Q)$ is said to be regular at $\left(x_{0}, f\right)$ if the following conditions are satisfied:

(1) We set $\theta^{l}=\left(\theta_{1}^{l}, \cdots, \theta_{m}^{l}\right)$. Then $p^{k} \theta^{l}$ is of rank constant on a neighborhood $\mathcal{Q}^{l+k} \subset \tilde{Q}^{l+k}$ of $j_{x_{0}}^{l+k}(f)$ for each $k \geqslant 0$. 
(2) We set $\mho^{k}=p^{k} \theta^{l}\left(\mathcal{Q}^{l+k}\right), k=0,1,2, \cdots$. For a map $\lambda$ of a neighborhood $\tilde{\mathcal{V}}$ of $x_{0}$ to $\mathbf{R}^{m}$ such that $j_{x}^{k}(\lambda) \in \mathcal{Q}^{k}$ for any $x \in \tilde{\mathcal{V}}$ and any integer $k \geqslant 0$, there exists a map $s$ of a neighborhood $\mathcal{V} \subset \tilde{\mathcal{V}}$ of $x_{0}$ to $Q$ such that $\theta^{l}\left(j_{x}^{l}(s)\right)=\lambda(x)$ on $\mathfrak{T}$.

Proposition 2.3.1. Let $\Theta^{l}=\left\{\theta_{j}^{l}\right\}_{J=1}^{m}$ be a family of functions at $j_{x_{0}}^{l}(f) \in$ $\tilde{J}^{l}(N, Q)$ which is regular at $\left(x_{0}, f\right)$. Then we have an integer $K$ and $a$ submanifold $W^{K}\left(\theta^{l}\right) \subset J^{K}\left(N, \mathbf{R}^{m}\right)$ satisfying the following conditions:

(1) $\mathcal{W}^{K}\left(\theta^{l}\right) \ni p^{K} \theta^{l}\left(j_{x_{0}}^{l+K}(f)\right)$ and $\left(\mathcal{Q S}^{K}\left(\theta^{l}\right), \alpha^{K}\left(\mathcal{W}^{K}\left(\theta^{l}\right)\right), \alpha^{K}\right)$ is a fibred manifold, where $\alpha^{K}$ is the projection of $J^{K}\left(N, \mathbf{R}^{m}\right)$ onto $N$.

(2) For a local map $\lambda$ of $N$ to $\mathbf{R}^{m}$, the differential equation $\theta^{l}=\lambda$ possesses $a$ solution if and only if $\lambda$ is a local cross secton of $\left(\mho^{K}\left(\theta^{l}\right), \alpha^{K}\left(\mho^{K}\left(\theta^{l}\right)\right) \alpha^{k}\right)$.

Proof. By the regularity condition (1) of $\Theta^{l}$ at $\left(x_{0}, f\right), \mathcal{V}^{k}=p^{k} \theta^{l}\left(\mathcal{Q}^{l+k}\right)$ is a submanifold of $J^{k}\left(N, \mathbf{R}^{m}\right)$ and $\left(\vartheta^{l+k}, \mho^{k}, p^{k} \theta^{l}\right)$ is a fibred manifold. Moreover it is clear that $\left(\mathcal{W}^{k}, \alpha^{k}\left(\mathcal{W}^{k}\right), \alpha^{k}\right)$ is also a fibred manifold. Since there is a neighborhood $\mathcal{U}^{l+k} \supset^{\prime} \mathcal{U}^{l+k}$ of $j_{x_{0}}^{l+k}(f)$ (resp. $\mathcal{U}^{l+k+1} \supset^{\prime} \mathcal{U}^{l+k+1}$ of $\left.j_{x_{0}}^{l+k+1}(f)\right)$ such that ( $\left(U^{l+k+1},{ }^{\prime+} U^{l+k}, \rho_{l+k}^{l+k+1}\right)$ is a fibred manifold and since $\left(\mathcal{Q}^{l+k+1}, \mathcal{W}^{k+1}, p^{k+1} \theta^{l}\right)$ and $\left(\mathcal{U}^{l+k}, \mathcal{W}^{k}, p^{k} \theta^{l}\right)$ are fibred manifolds, there exist neighborhoods $\mho^{k+1} \supset^{\prime} W^{k+1}$ of $p^{k+1} \theta^{l}\left(j_{x_{0}}^{l+k+1}(f)\right)$ and $\mho^{k} \supset$ 'थ $w^{k}$ of $p^{k} \theta^{l}\left(J_{x_{0}}^{l+k}(f)\right)$ such that $\left(' W^{1+k},{ }^{\prime} W^{k}, \rho_{k}^{k+1}\right)$ is a fibred manifold. It is clear that we have $p \mho^{k} \supset \mathcal{Q ⿻}^{k+1}$ and $\theta^{l}\left(j^{l}(f)\right)$ is a solution of $\mho^{k}$ for any $k \geqslant 0$. Therefore by Kuranishi's prolongation theorem, there exists an integer $K$ such that, for any $k \geqslant K$, $\mho^{k}$ is involutive at $p^{k} \theta^{l}\left(j_{x_{0}}^{l+k}(f)\right)$ and $p \mho^{k}=$ Q $^{k+1}$ on a neighborhood of $p^{k+1} \theta^{l}\left(j_{x_{0}}^{l+k+1}(f)\right)$. We set ข $^{K}\left(\theta^{l}\right)=$ Q $^{K}$. Let $\lambda$ be a solution of $\mathscr{W}^{K}\left(\theta^{l}\right)$. Then $\lambda$ is also a solution of $\mho^{k}$ for any $k>K$. Therefore, by the regularity condition (2) of $\Theta^{l}$ at $\left(x_{0}, f\right)$, for a fixed $\tilde{x}_{0} \in N$, we have a local map $s$ of $N$ to $Q$ such that $j_{\tilde{x}_{0}}^{\alpha}\left(\theta^{l}\left(j^{l}(s)\right)\right)=j_{\tilde{x}_{0}}^{\alpha}(\lambda)$ for any integer $\alpha \geqslant 0$. This implies that if $\lambda$ is a local cross secton of $\left(\mathcal{W}^{K}\left(\theta^{l}\right)\right.$, $\left.\alpha^{K}\left(\mathscr{W}^{K}\left(\theta^{l}\right)\right), \alpha^{K}\right)$, then the differential equation $\theta^{l}=\lambda$ possesses a solution. Clearly if $\theta^{l}=\lambda$ possesses a solution, $\lambda$ is a local cross section of ( $\mathcal{W}^{K}\left(\theta^{l}\right)$, $\left.\alpha^{K}\left(\mho^{K}\left(\theta^{l}\right)\right), \alpha^{K}\right)$. This completes the proof of Proposition 2.3.1.

Definition 2.3.2. The space $\mho^{K}\left(\theta^{l}\right)$ in Proposition 4.1 is called a resolvent space of $\theta^{l}$. It is clear that if $\mathcal{Q S}^{K}\left(\theta^{l}\right)$ is a resolvent space of $\theta^{l}$, we have a resolvent space $\mathcal{Q S}^{H}\left(\theta^{l}\right)$ of $\theta^{l}$ for any integer $H \geqslant K$.

Definition 2.3.3. Let $\Theta^{l}=\left\{\theta_{j}^{l}\right\}_{j=1}^{h}$ be a family of functions at $j_{x_{0}}^{l}(f)$ which is regular at $\left(x_{0}, f\right)$ and let $\mho^{K}\left({ }^{l}\right)=p^{K} \theta^{l}\left(\mathcal{Q}^{l+K}\right)$ be any resolvent space of $\theta^{l}$. We set $\mathcal{Q}^{K}\left(\theta^{l}, E\right)=p^{K} \theta^{l}\left(I\left(p^{l+K-\alpha}(E)\right) \cap \mathcal{Q}^{l+K}\right)$, where $p^{l+K-\alpha}(E)$ is the $(l+K-\alpha)$-th prolongation of a differential equation $E$ at $j_{x_{0}}^{\alpha}(f)$. $\mho^{K}\left(\theta^{l}, E\right)$ is called a resolvent space of $\left(\theta^{l}, E\right) . \Theta^{l}$ is said to be $E$-regular at $\left(x_{0}, f\right)$ if $\mathcal{W}^{K}\left(\theta^{l}, E\right)$ is a regular submanifold of $\mathcal{W}^{K}\left(\theta^{l}\right)$ for a neighborhood $\mathcal{Q}^{l+K}$ and 
any integer $K \geqslant K_{0}$ where $K_{0}$ is the minimum integer for which $\theta^{l}$ possesses a resolvent space.

Proposition 2.3.2. Let $\Theta^{l}=\left\{\theta_{j}^{l}\right\}_{j=1}^{h}$ be a family of functions at $j_{x_{0}}^{l}(f) \in$ $\tilde{J}^{l}(N, Q)$ which is regular at $\left(x_{0}, f\right)$. Let $E$ be an involutive differential equation at $j_{x_{0}}^{\alpha}(f), \alpha \leqslant l$. Suppose that $\Theta^{l}$ is $E$-regular at $\left(x_{0}, f\right)$ and that, for any solution $\lambda$ of the resolvent space $W^{K}\left(\theta^{l}, E\right)$ of $\left(\theta^{l}, E\right)$, we have $I\left(\theta^{l}(\lambda)\right) \subset I\left(p^{l-\alpha}(E)\right)$, where $\theta^{l}(\lambda)$ is the differential equation generated by $\theta_{j}^{l}-\lambda_{j}(1<j \leqslant h)$. Then there exists a generator $\mathcal{H}=\left\{\mathfrak{h}_{j}\right\}_{j=1}^{r}$ of $p^{\beta}(E), \beta=l+K-\alpha$, such that $\mathfrak{h}_{j}$ is of the form $\left(p^{K} \theta^{l}\right)^{*} F_{j}$, where $F_{j}$ is a function at $p^{K} \theta^{l}\left(j_{x_{0}}^{l+k}(f)\right) \in J^{l+K}\left(N, \mathbf{R}^{h}\right)$, $1<j \leqslant r$.

Proof. Let $\left\{F_{1}, \cdots, F_{r}\right\}$ be a family of linearly independent functions at $p^{K} \theta^{l}\left(j_{x_{0}}^{l+K}(f)\right)=j_{x_{0}}^{K}\left(\theta^{l}(f)\right)$ by which $\mho^{K}\left(\theta^{l}, E\right)$ is locally defined at $j_{x_{0}}^{K}\left(\theta^{l}(f)\right)$. We set $\mathfrak{h}_{j}=\left(p^{K} \theta^{l}\right)^{*} F_{j}(1 \leqslant j \leqslant r)$. We denote by $(*)$ the differential equation generated by $\left\{\mathfrak{h}_{j}\right\}_{j=1}^{r}$. Then it is clear that $\delta(*) \supset \mathcal{S}\left(p^{\beta}(E)\right)$. On the other hand by the assumption, we have $I\left(\theta^{l}(\lambda)\right) \subset I\left(p^{l-\alpha}(E)\right)$ for any solution $\lambda$ of $\mho^{K}\left(\theta^{l}, E\right)$. This implies that $\delta(*) \subset \delta\left(p^{\beta}(E)\right)$. Therefore we get $\delta(*)=$ $\delta\left(p^{\beta}(E)\right)$. Then it is easy to see that $I(*)=I\left(p^{\beta}(E)\right)$ because $E$ is involutive and the resolvent space $\mathscr{W}^{K}\left(\theta^{l}, E\right)$ is defined by $F_{1}, \cdots, F_{r}$. This means that $p^{\beta}(E)$ is generated by $\left\{\mathfrak{h}_{j}\right\}_{j=1}^{r}$. The proof is completed.

Definition 2.2.4. A differential equation $E$ at $j_{x}^{k}(f) \in \tilde{J}^{k}(N, Q)$ is said to be $\Gamma$-closed if the automorphism pseudo-group $\mathcal{Q}(E)$ of $E$ is equal to $\Gamma$ on a neighborhood of $f(x)$.

Corollary 2.2.1. Suppose $\mathfrak{L}_{\Gamma}$ is an $N$-regular weak Lie algebra sheaf and let $\Theta^{l}=\left\{\theta_{j}^{l}\right\}_{j=1}^{m_{l}}$ be a fundamental system of differential invariants of $\mathcal{E}_{T}$ at $j_{x_{0}}^{l}(f)$. Let $E$ be a $\Gamma$-closed involutive differential equation at $j_{x_{0}}^{\alpha}(f)$, where $l+K-\alpha$ $\geqslant 0$. Suppose $\Theta^{l}$ is regular and E-regular at $\left(x_{0}, f\right)$. Then there exists a $\Gamma$-family $\mathcal{C}=\left\{\mathfrak{h}_{j}\right\}_{j=1}^{r}$ at $j_{x_{0}}^{l+K}(f)$ of type $(l, r)$ such that $\mathcal{H}$ is a generator of $p^{\beta}(E)$ at $j_{x_{0}}^{l+K}(f), \beta=l+K-\alpha$.

Proof. Since $E$ is $\Gamma$-closed, it is easy to see that, for any solution $\lambda$ of $\mho^{K}\left(\theta^{l}, E\right)$ we have $I\left(\theta^{l}(\lambda)\right) \subset I\left(p^{l-\alpha}(E)\right)$, where $\theta^{l}(\lambda)$ is the $\mathscr{L}_{T}$-orbit system generated by $\theta_{j}^{l}-\lambda_{j}\left(1 \leqslant j \leqslant m_{l}\right)$. Therefore, by Proposition 2.3.2, $p^{\beta}(E)$ possesses a generator $\mathcal{H}=\left\{\mathfrak{h}_{j}\right\}_{j=1}^{r}$ such that $\mathfrak{h}_{j}$ is a $\Gamma$-differential invariant. It is clear that $\mathcal{H}=\left\{\mathfrak{h}_{j}\right\}_{j=1}^{r}$ is a $\Gamma$-family at $j_{x_{0}}^{l+K}(f)$ of type $(l, r)$.

\section{Reduction of equivalence}

3.1. Let $\Gamma$ be a pseudo-group on $Q$, such that $\varrho_{\Gamma}$ is an $N$-regular weak Lie algebra sheaf. For a fundamental system of differential invariants $\Theta^{l}=$ 
$\left\{\theta_{j}^{l}\right\}_{j=1}^{m_{l}}$ of $\mathscr{L}_{\Gamma}$ at $j_{x_{0}}^{l}(f) \in \tilde{J}^{l}(N, Q)$, there are neighborhoods $\mathcal{Q}^{l}$ of $j_{x_{0}}^{l}(f)$ and ขf of $\theta^{l}\left(j_{x_{0}}^{l}(f)\right) \in \mathbf{R}^{m_{l}}$ such that $\left(\mathcal{Q}^{l}, \mathcal{W}, \boldsymbol{\theta}^{l}\right)$ is a fibred manifold. Let $\Re(\Gamma)$ be the normalizer of $\Gamma$ in the pseudo-group of all local diffeomorphisms of $Q$. Then, for $\phi^{(l)} \in(\Re(\Gamma))^{(l)} \mid \mathcal{Q}^{l}$, we can induce a local diffeomorphism $D\left(\theta^{l}, \phi\right)$ of $\mathscr{W}$ such that $\theta^{l} \circ \phi^{(l)}=D\left(\theta^{l}, \phi\right) \circ \theta^{l}$. We denote by $D\left(\theta^{l}, \Re(\Gamma)\right)$ or simply by $D(\Re(\Gamma))$ the pseudo-group on $\mathscr{T}$ generated by $D\left(\theta^{l}, \phi\right), \phi \in$

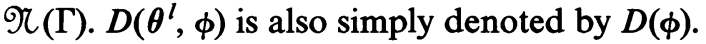

Let $E^{1}$ and $E^{2}$ be $\Gamma$-closed differential equations at $j_{x_{0}}^{\alpha^{1}}(f)$ and at $j_{x_{0}}^{\alpha^{2}}(f)$ respectively. Suppose there is an integer $K$ such that $l+K-\alpha^{i}=\beta^{i} \geqslant 0$, $i=1,2$, and such that $p^{\beta^{i}}\left(E^{i}\right)$ possesses a generator $F^{i}\left(\theta^{l}\right)=\left\{F_{j}^{i}\left(\theta^{l}\right)\right\}_{j=1}^{r}$ which is a $\Gamma$-family at $j_{x_{0}}^{l+K}(f)$ of type $(l, r)$. We denote by $D^{K}\left(\theta^{l}, E^{i}\right)$ or simply by $D^{K}\left(E^{i}\right)$ the differential equation at $p^{K} \theta^{l}\left(j_{x_{0}}^{l+K}(f)\right)$ generated by $\left\{F_{!}^{i}, \cdots, F_{r}^{i}\right\}$.

Proposition 3.1.1. $E^{1}$ is isomorphic to $E^{2}$ if and only if there is an element $\phi \in \Re(\Gamma)$ such that $D(\phi)$ is an isomorphism of $D^{K}\left(E^{1}\right)$ to $D^{K}\left(E^{2}\right)$.

Proof. Let $\phi$ be an isomorphism of $E^{1}$ to $E^{2}$. Since $\mathcal{Q}\left(E^{i}\right)=\Gamma$ on a neighborhood of $f\left(x_{0}\right)$, it is easy to see that $\phi \in \mathcal{N}(\Gamma)$. Let $\lambda^{1}$ be a solution of $D^{K}\left(E^{1}\right)$ and consider the differential equation $\theta^{l}=\lambda^{1}$. We denote by $\delta\left(\lambda^{1}\right)$ the solution space of the equation. Then, since $p^{\beta^{i}}\left(E^{i}\right)$ possesses as a generator a $\Gamma$-family $F^{i}\left(\theta^{l}\right)$ at $j_{x_{0}}^{l+K}(f)$ of type $(l, r)$ it is clear that $\delta\left(E^{i}\right)=$ $\cup_{\lambda^{i} \in \delta\left(D^{K}\left(E^{i}\right)\right)} \delta\left(\lambda^{i}\right)$, where $\delta\left(\lambda^{i}\right) \cap \delta\left(\mu^{i}\right) \neq \varnothing$ if and only if $\delta\left(\lambda^{i}\right)=$ $\mathcal{S}\left(\mu^{i}\right)$. Since $\phi \in \Re(\Gamma), \phi^{(l) *} \theta^{l}=\xi\left(\theta^{l}\right)$. Therefore $\theta^{l}\left(j_{x}^{l}(\phi \circ s)\right)=$

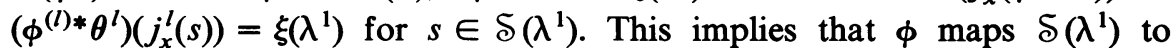
$\mathcal{S}\left(\lambda^{2}\right)$, where $\lambda^{2}=\xi\left(\lambda^{1}\right)$, that is, for each $\lambda^{1} \in \mathcal{S}\left(D^{K}\left(E^{1}\right)\right)$, there corresponds $\lambda^{2} \in \mathcal{S}\left(D^{K}\left(E^{2}\right)\right)$ such that $\lambda^{2}=D(\phi) \circ \lambda^{1}$ and such that the isomorphism $\phi$ of $E^{1}$ to $E^{2}$ maps $\delta\left(\lambda^{1}\right)$ to $\delta\left(\lambda^{2}\right)$. This means that $D(\phi)$ is an isomorphism of $D^{K}\left(E^{1}\right)$ to $D^{K}\left(E^{2}\right)$. Conversely, assume that there is an element $\phi \in \Re(\Gamma)$ such that $D(\phi)$ is an isomorphism of $D^{K}\left(E^{1}\right)$ to $D^{K}\left(E^{2}\right)$. Then, for $s \in$ $\mathcal{S}\left(E^{1}\right)$, if $s \in \mathcal{S}\left(\lambda^{1}\right)$, we have $\theta^{l}\left(j_{x}^{l}(\phi \circ s)\right)=\phi^{(l) *} \theta^{l}\left(j_{x}^{l}(s)\right)=D(\phi) \circ \theta^{l}\left(j_{x}^{l}(s)\right)$ $=D(\phi) \circ \lambda^{1}=\lambda^{2} \in \mathcal{S}\left(D^{K}\left(E^{2}\right)\right)$. Therefore $\phi \circ s \in \mathcal{S}\left(E^{2}\right)$. This means that $\phi$ is an isomorphism of $E^{1}$ to $E^{2}$. This completes the proof of Proposition 3.1.1.

Remark 3.3.1. Let $E$ be a differential equation at $j_{x}^{\alpha}(f) \in \tilde{J}^{\alpha}(N, Q)$. Then, roughly speaking, Corollary 2.2 .1 implies that $E$ is a family $\mathscr{F}$ of $\mathscr{Q}(E)$-orbit systems, where $\mathscr{F}$ is parametrized by the solution space of a differential equation $D^{K}(E)$. That is, the structure of $E$ is decomposed into that of $\mathcal{Q}(E)$-orbit systems and that of $D^{K}(E)$. Proposition 3.1.1 implies that the equivalence problem of $\Gamma$-closed differential equations $E_{1}$ and $E_{2}$ is reduced to that of $D^{K}\left(E_{1}\right)$ and $D^{K}\left(E_{2}\right)$ under the pseudo-group $D(\Re(\Gamma))$. 


\section{Reducibility of differential equations}

4.1. Definition 4.1.1. Let $\left(Q, Q^{\prime}, \pi\right)$ be a fibred manifold. A differential equation $E$ at $j_{x_{0}}^{\alpha}(f) \in J^{\alpha}(N, Q)$ is said to be weakly $K$-reducible to a differential equation $E^{\prime}$ at $j_{x_{0}}^{\alpha^{\prime}}(\pi \circ f) \in J^{\alpha^{\prime}}\left(N, Q^{\prime}\right)$ by $\pi$ if there exist a nonnegative integer $K$ and, for $k \geqslant K$, neighborhoods $\mathcal{Q}^{k}$ and $\mathcal{Q}^{\prime k}$ of $j_{x_{0}}^{k}(f)$ and $j_{x_{0}}^{k}(\pi \circ f)$, respectively, satisfying the following conditions:

(1) $\left(\mathcal{Q}^{k}, \mathcal{U}^{\prime k}, \pi^{k}\right)$ is a fibred manifold, where $\pi^{k}$ is the map naturally induced from $\pi$.

(2) (i) For any $s \in \delta(E) \mid \mathcal{Q}^{k}$, we have $\pi \circ s \in \delta\left(E^{\prime}\right) \mid \mathcal{Q}^{\prime k}$. (ii) For any $s^{\prime} \in \mathcal{S}\left(E^{\prime}\right) \mid \mathcal{U}^{\prime k}$, we have an $s \in \mathcal{S}(E) \mid \mathcal{Q}^{k}$ with $s^{\prime}=\pi \circ s$. (iii) If $s: U \rightarrow V$ or $\tilde{s}: \tilde{U} \rightarrow \tilde{V}$ belongs to $\delta(E) \mid \mathcal{Q}^{k}$ with $\pi \circ s=\pi \circ \tilde{s}$ on $U \cap \tilde{U}$, then we have $s=\tilde{s}$ on $U \cap \tilde{U}$, where $\mathcal{S}(E) \mid \mathcal{Q}^{k}=\left\{g \in \mathcal{S}(E) ; \operatorname{Im} j^{k}(g) \subset \mathcal{Q}^{k}\right\}$.

Furthermore if $E$ satisfies the following condition (3), $E$ is said to be $K$-reducible to $E^{\prime}$ by $\pi$ : We set $\left.\mathbb{Q}(E)\right|^{\mathcal{Q}^{k}}=\left\{g \in \mathbb{Q}(E) ;{ }^{\sharp} \psi \in \mathbb{Q}(E)^{(k)} \mid \mathcal{Q}^{k}\right.$ such that $\left.g \circ \beta^{k}=\beta^{k} \circ \psi\right\}$. Then

(3) For any $g: U \rightarrow V$ which belongs to $\mathcal{Q}(\mathrm{E}) \mid \mathcal{Q}^{k}$, there is a diffeomorphism $g^{\prime}$ of $\pi(U)$ to $\pi(V)$ such that $g^{\prime} \circ \pi=\pi \circ g$ for $k \geqslant K$.

Then we denote by $\mathcal{Q}(E)_{k}^{\prime}$ the pseudo-group on a neighborhood of $f\left(x_{0}\right)$ generated by $\left\{g^{\prime} ; g \in \mathbb{Q}(E) \mid Q^{k}, \pi \circ g=g^{\prime} \circ \pi\right\}$.

We say that $E^{\prime}$ is a (weakly) $K$-reduced form of $E$ and the pair $\left(E, E^{\prime}\right)$ is called a (weakly) $K$-reduced pair.

Let $E$ be a differential equation at $j_{x_{0}}^{l}(f) \in \tilde{J}^{l}(N, Q)$. For a neighborhood $\mathcal{Q}^{l}$ of $j_{x_{0}}^{l}(f)$, we set $S\left(E, \mathcal{Q}^{l}\right)=\cup_{s \in \delta(E)} \operatorname{Im} j^{l}(s) \cap \mathcal{Q}^{l}$.

Corollary 2.2.1 makes the following definition significant.

Definition 4.1.2. A differential equation $E$ at $j_{x}^{\alpha}(f) \in \tilde{J}^{\alpha}(N, Q)$ is said to be $l$-regular at $x$ if $E$ satisfies the following conditions (1), (2) and (3):

(1) The sheaf $\mathfrak{E}_{\mathfrak{Q}(E)}$ associated to the automorphism pseudo-group $\mathcal{Q}(E)$ is an $N$-regular weak Lie algebra sheaf on a neighborhood of $f(x)$.

(2) There are an integer $K^{\tilde{l}}$ and an $\mathcal{Q}(E)$-family $\mathcal{H}^{\tilde{l}}=\left\{\mathfrak{h}_{j}^{\tilde{l}}\right\}_{j=1}^{r}$ at $j_{x}^{i+K^{i}}(f)$ of type $(\tilde{l}, r)$ such that $\tilde{l}+K^{\tilde{l}}-\alpha \geqslant 0$ and $p^{\tilde{l}+K^{i}-\alpha}(E)$ is generated by $\mathcal{H C}^{\tilde{l}}$ for $\tilde{l} \geqslant l$.

(3) There is a neighborhood $\mathcal{Q}^{k}$ of $j_{x}^{k}(f)$ such that $S\left(E, \mathcal{Q}^{k}\right)$ is a regular submanifold of $\tilde{J}^{k}(N, Q)$ for $k \geqslant l$.

We denote by $D^{K^{i}}\left(\theta^{i}, E\right)$ the differential equation generated by $F_{1}^{\tilde{l}}, \cdots, F_{r}^{\tilde{l}}$ where $\mathfrak{h}_{j}^{\tilde{l}}=F_{j}^{\tilde{l}}\left(\theta^{\tilde{l}}\right)$. It is called a resolvent system of $E$. (Refer to Definition 2.3.3.)

Remark 4.1.1. If a differential equation $E$ at $j_{x}^{\alpha}(f)$ is $l$-regular at $x$, then it is clear that $E$ is $k$-regular at $x$ for any $k \geqslant l$.

Let $\Gamma^{1}$ and $\Gamma^{2}$ be pseudo-groups on $Q$, and $j_{x}^{l}(f)$ a point of $\tilde{J}^{l}(N, Q)$. We 
say that $\Gamma^{1}=\Gamma^{2}$ on a neighborhood $\mathcal{Q}^{1}$ of $j_{x}^{l}(f)$ if $\Gamma^{1}\left|\mathcal{U}^{l}=\Gamma^{2}\right| \mathcal{U}^{l}$.

Definition 4.1.3. Let $\Gamma$ be a pseudo-group on $Q$ such that $\varrho_{\Gamma}$ is an $N$-regular weak Lie algebra sheaf. $\Gamma$ is said to be $\tilde{l}$-closed at $(x, f)$ if there is an integer $\tilde{l}$ such that for $l \geqslant \tilde{l}, \mathbb{Q}\left(\mathfrak{E}_{\Gamma}(l, x, f)\right)=\Gamma$ on a neighborhood of $j_{x}^{l}(f)$, where $\mathfrak{E}_{T}(l, x, f)$ is the $\mathfrak{C}_{\Gamma}$-orbit system at $(l, x, f)$.

Remark 4.1.2. When $\operatorname{dim} N \geqslant \operatorname{dim} Q$, in Lemma 5.1.2 we shall show that any pseudo-group which is complete at $\left(z_{0}, 1\right)$ is l-closed at $\left(x_{0}, f\right)$, where $z_{0}=f\left(x_{0}\right)$, and $l$ is the order of the pseudo-group at $\left(x_{0}, f\right)$. When $\operatorname{dim} N<$ $\operatorname{dim} Q$, in $\$ 7$ we shall give an example of a pseudo-group which is closed at $\left(x_{0}, f\right)$ where $f$ is a local immersion of $N$ to $Q$.

Let $f$ be a local map of a neighborhood of $x \in N$ to $Q$ which is an immersion (resp. a submersion) if $\operatorname{dim} N<\operatorname{dim} Q($ resp. $\operatorname{dim} N \geqslant \operatorname{dim} Q)$.

Definition 4.1.4. A pseudo-group $\Gamma$ on $Q$ is said to be $l$-automorphic at $(x, f)$ where $l$ is a nonnegative integer, if the following conditions are satisfied:

(1) $\mathscr{L}_{T}$ is an $N$-regular weak Lie algebra sheaf.

(2) The orbit system $\varrho_{T}(l, x, f)$ is $\Gamma$-automorphic.

As for the definition of " $\Gamma$-automorphic system", refer to Definition 9.1.2.

Let $\left(Q, Q^{\prime}, \pi\right)$ be a fibred manifold, and let $E$ (resp. $\left.E^{\prime}\right)$ be a differential equation at $j_{x_{0}}^{\alpha}(f) \in \tilde{J}^{\alpha}(N, Q)$ (resp. $j_{x_{0}}^{\alpha^{\prime}}(\pi \circ f) \in \tilde{J}^{\alpha^{\prime}}\left(N, Q^{\prime}\right)$ ). Suppose $E$ and $E^{\prime}$ are $l$-regular at $x_{0}$, and let $\left\{\theta_{j}^{k}\right\}_{j=1}^{m_{k}}$ (resp. $\left\{\theta_{j}^{\prime k}\right\}_{j=1}^{m_{k}^{\prime}}$ ) be a fundamental system of differential invariants of $\mathcal{E}_{\mathscr{Q}(E)}$ at $j_{x_{0}}^{k}(f)$ (resp. $\mathcal{L}_{\mathscr{Q}_{\left(E^{\prime}\right)}}$ at $j_{x_{0}}^{k}(\pi \circ f)$ ). We denote by $D_{E}^{k}$ (resp. $D_{E^{\prime}}^{k}$ ) the involutive distribution on a neighborhood of $j_{f\left(x_{0}\right)}^{k}(1) \in \tilde{J}^{k}(Q, Q)$ (resp. $\left.j_{\pi \circ f\left(x_{0}\right)}^{k}(\pi \circ 1) \in \tilde{J}^{k}\left(Q, Q^{\prime}\right)\right)$ induced from $\mathcal{L}_{\mathcal{Q}(E)}$ (resp. $\mathcal{L}_{\left.\mathcal{Q}_{\left(E^{\prime}\right)}\right)}$.

Lemma 4.1.1. Suppose $E$ is $K$-reducible to $E^{\prime}$ by $\pi$. Then the following two assertions (A) and (B) hold.

(A) For any $\tilde{l} \geqslant l^{\prime}=\max (l, K)$, we have a fibred manifold $\left(\mathcal{\Theta}^{i}, \mathcal{O}^{\prime i}, D^{i}(\pi)\right)$ satisfying the following properties:

(1) $\theta^{\tilde{l}}$ of $\theta^{\prime i}$ is a neighborhood of $\boldsymbol{\theta}^{\tilde{l}}\left(j_{x_{0}}^{\tilde{l}}(f)\right) \in \mathbf{R}^{m_{i}}$ or $\theta^{\prime i}\left(j_{x_{0}}^{i}(\pi \circ f)\right) \in \mathbf{R}^{m_{i}^{i}}$, respectively.

(2) $D^{\tilde{i}}(\pi)$ induces an onto map $O_{E}^{\tilde{i}}$ of $\delta\left(D^{K^{i}}\left(\theta^{i}, E\right)\right) \mid \mho^{K^{i}}$ to

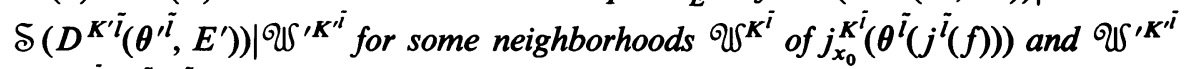
of $j_{x_{0}}^{K^{i}}\left(\theta^{\prime i}\left(j i{ }^{i}(\pi \circ f)\right)\right)$.

(3) For any $\lambda \in \mathcal{S}\left(D^{K^{i}}\left(\theta^{i}, E\right)\right) \mid \mathscr{W}^{K^{i}}$, the space $\mathcal{S}\left(\theta^{i}(\lambda)\right)$ is transferred by $\pi$ into $\mathcal{S}\left(\theta^{\prime i}\left(\lambda^{\prime}\right)\right)$, where $\lambda^{\prime}=D^{i}(\pi) \circ \lambda, \theta^{i}(\lambda)$ is an $\mathfrak{L}_{\mathscr{Q}(E)}$-orbit system generated by $\theta_{j}^{\tilde{l}}-\lambda_{j}\left(1 \leqslant j \leqslant m_{i}\right)$, and $\theta^{\prime i}\left(\lambda^{\prime}\right)$ is an $\mathcal{L}_{\mathfrak{Q}\left(E^{\prime}\right)}$-orbit system generated by $\theta_{j}^{\prime l}-\lambda_{j}^{\prime}\left(1 \leqslant j \leqslant m_{l}^{\prime}\right)$.

(B) If one of the following two conditions holds, $\theta^{k}(\lambda)$ is weakly $k$-reducible to 
$\theta^{\prime k}\left(\lambda^{\prime}\right)$ by $\pi$ for $k \geqslant l^{\prime}$, where $\lambda=\theta^{k}\left(j^{k}(f)\right)$, and $\lambda^{\prime}=D^{k}(\pi) \circ \lambda$ :

(i) The onto map $O_{E}^{k}$ is one to one.

(ii) $\operatorname{dim}\left(\pi^{k}\right)_{*} D_{E}^{k}=\operatorname{dim} D_{E^{\prime}}^{k}, \mathcal{Q}\left(E^{\prime}\right)$ and $\mathbb{Q}(E)_{k}^{\prime}$ are complete at $\left(\pi \circ f\left(x_{0}\right), 1\right)$, and $\mathbb{Q}\left(E^{\prime}\right)$ is $k$-automorphic at $\left(x_{0}, \pi \circ f\right)$ for a sufficiently large integer $k \geqslant l^{\prime}$. Moreover, if $\mathcal{Q}(E)$ is $k$-closed at $\left(x_{0}, f\right)$, then $\theta^{k}(\lambda)$ with $\lambda=\theta^{k}\left(j^{k}(f)\right)$ is $k$-reducible to $\theta^{\prime k}\left(\lambda^{\prime}\right)$ with $\lambda^{\prime}=D^{k}(\pi) \lambda$ by $\pi$.

Proof. Let $g \in \mathbb{Q}(E) \mid \mathcal{Q}^{l^{\prime}}$. Then we have a local diffeomorphism $g^{\prime}$ of $Q^{\prime}$ such that $g^{\prime} \circ \pi=\pi \circ g$. Let $s^{\prime} \in \delta\left(E^{\prime}\right) \mid \mathcal{Q}^{\prime \prime^{\prime}}$, and set $\bar{s}^{\prime}=g^{\prime} \circ s^{\prime}$. Since $E$ is $l^{\prime}$-reducible to $E^{\prime}$ by $\pi$, we have an $s \in \delta(E) \mid \mathcal{Q l}^{l^{\prime}}$ such that $s^{\prime}=\pi \circ s$. Therefore we get $\bar{s}^{\prime}=g^{\prime} \circ s^{\prime}=g^{\prime} \circ \pi \circ s=\pi \circ g \circ s$. Since $g \in \mathbb{Q}(E) \mid \mathcal{U}^{l^{\prime}}$ and $s \in \mathcal{S}(E) \mid \mathcal{Q}^{l^{\prime}}$, we have $g \circ s \in \mathcal{S}(E) \mid \mathcal{Q l}^{l^{\prime}}$. Thus we get $\bar{s}^{\prime}=\pi \circ g \circ s \in$ $\delta\left(E^{\prime}\right) \mid \mathcal{U}^{\prime l^{\prime}}$ because of the $l^{\prime}$-reducibility of $E$ to $E^{\prime}$ by $\pi$. Since $s^{\prime}$ is any element of $\delta\left(E^{\prime}\right) \mid \mathcal{Q}^{l^{\prime}}$, we get $g^{\prime} \in \mathbb{Q}\left(E^{\prime}\right) \mid \mathcal{Q}^{\prime l^{\prime}}$.

We set $\eta_{j}^{i}=\left(\pi^{\tilde{l}}\right)^{*} \theta_{j}^{\prime \tilde{l}}\left(1 \leqslant j \leqslant m_{l}^{l}\right)$. Then, by the above stated fact, $\eta_{j}^{i}$ $\left(1<j \leqslant m_{l}^{l}\right)$ is a linearly independent differential invariant of $\mathcal{L}_{\mathscr{Q}(E)}$ at $j_{x_{0}}^{i}(f)$. Let $\left\{\eta_{j}^{i}\right\}_{j=1}^{m_{i}}$ be a fundamental system of differential invariants of $\mathcal{E}_{\Theta(E)}$ at $j_{x_{0}}^{i}(f)$. Then we have expressions $\eta_{j}^{i}=\phi_{j}^{i}\left(\theta_{1}^{i}, \cdots, \theta_{m_{i}}^{i}\right)\left(1<j \leq m_{i}\right)$. For $s_{1}$ and $s_{2} \in \mathcal{S}\left(\theta^{i}(\lambda)\right)$, if we set $s_{i}^{\prime}=\pi \circ s_{i}$, we have $\theta_{j}^{\prime i}\left(j_{x}^{i}\left(s_{i}^{\prime}\right)\right)=\theta_{j}^{\prime i}\left(j_{x}^{i}\left(\pi \circ s_{i}\right)\right)=$ $\left(\pi^{\tilde{l}}\right)^{*} \theta_{j}^{\prime i}\left(j_{x}^{\tilde{l}}\left(s_{i}\right)\right)=\eta_{j}^{\tilde{l}}\left(j_{x}^{\tilde{l}}\left(s_{i}\right)\right)$. Since $\theta_{j}^{\tilde{l}}\left(j_{x}^{\tilde{l}}\left(s_{1}\right)\right)=\theta_{j}^{\tilde{l}}\left(j_{x}^{\tilde{l}}\left(s_{2}\right)\right)=\lambda_{j}(x)\left(1<j<m_{i}\right)$, we get $\theta_{j}^{\prime l}\left(j_{x}^{l}\left(s_{1}^{\prime}\right)\right)=\theta_{j}^{\prime l}\left(j_{x}^{l}\left(s_{2}\right)\right)=\lambda_{j}^{\prime}(x)=\phi_{j}^{i}\left(\lambda_{1}(x), \cdots, \lambda_{m_{i}}(x)\right)\left(1<j<m^{\prime}\right)$. This means that $\delta\left(\theta^{i}(\lambda)\right)$ is transferred into $\delta\left(\theta^{\prime i}\left(\lambda^{\prime}\right)\right)$ by $\pi$. Since $E$ is $K$-reducible to $E^{\prime}$ by $\pi$, we have a fibred manifold $\left(\mathcal{Q}^{k}, \mathcal{Q}^{\prime k}, \pi^{k}\right)$ such that $\mathcal{Q}^{k}$ (resp. $\left.\mathcal{Q}^{\prime k}\right)$ is a neighborhood of $j_{x_{0}}^{k}(f)$ (resp. $j_{x_{0}}^{k}(\pi \circ f)$ ) and such that $\pi$ induces a map of $\delta(E) \mid \mathcal{Q}^{k}$ onto $\delta\left(E^{\prime}\right) \mid \mathcal{Q}^{\prime k}, k \geqslant K$. Therefore, if we set

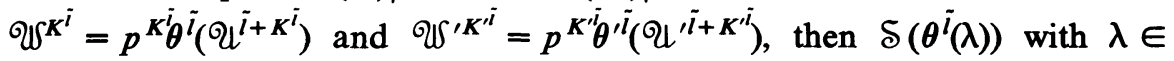
$\delta\left(D^{K^{i}}\left(\theta^{\tilde{i}}, E\right)\right) \mid \mho^{K^{i}}$ is transferred into $\delta\left(\theta^{\prime i}\left(\lambda^{\prime}\right)\right)$ for some $\lambda^{\prime} \in$ $\left.\delta\left(D^{K^{i}}\left(\theta^{\prime i}, E^{\prime}\right)\right)\right|^{\mathcal{W}^{\prime} K^{\prime i}}$. We set $\phi^{i}=\left(\phi_{1}^{i}, \cdots, \phi_{m_{i}}^{i}\right)$, and denote by $p$ the projection of $\mathbf{R}^{m_{i}}$ onto $\mathbf{R}_{i}^{m_{i}^{\prime}}$ defined by $p\left(x_{1}, \cdots, x_{m_{i}}\right)=\left(x_{1}, \cdots, x_{m_{i}}\right)$. We set

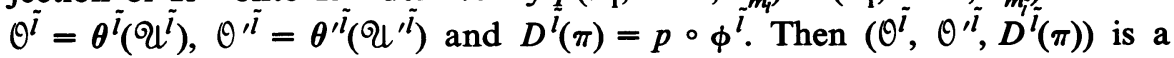
fibred manifold satisfying conditions (1), (2) and (3). This proves (A).

If the condition (i) is satisfied, it is now clear that $\theta^{k}(\lambda)$ is weakly $k$-reducible to $\theta^{\prime k}\left(\lambda^{\prime}\right)$ by $\pi$ for $k \geqslant l^{\prime}$.

Now we assume the condition (ii). Since $E$ is $K$-reducible to $E^{\prime}$, we see that $\left(\pi^{k}\right)_{*} D_{E}^{k}=D_{E^{\prime}}^{k}$ for a sufficiently large $k$. On the other hand, since $\mathcal{Q}\left(E^{\prime}\right)$ and $\mathcal{Q}(E)_{k}^{\prime}$ are complete at $\left(\pi \circ f\left(x_{0}\right), 1\right)$, by [6, Proposition 8.1] we can easily see that $\mathscr{Q}(E)_{k}^{\prime}\left|\mathcal{Q}^{\prime k}=\mathscr{Q}\left(E^{\prime}\right)\right|^{\prime \prime k}$ for a neighborhood $\mathcal{U}^{\prime k}$ of $j_{x_{0}}^{k}(\pi \circ f)$. Since $\mathcal{Q}\left(E^{\prime}\right)$ is $k$-automorphic at $\left(x_{0}, \pi \circ f\right), \theta^{\prime k}\left(\lambda^{\prime}\right)$ is $\mathscr{Q}(E)_{k}^{\prime}$-automorphic.

Since $\pi$ maps $\delta\left(\theta^{k}(\lambda)\right) \mid \mathcal{Q}^{k}$ into $\delta\left(\theta^{\prime k}\left(\lambda^{\prime}\right)\right) \mid \mathcal{U}^{\prime k}$, and since $\theta^{\prime k}\left(\lambda^{\prime}\right)$ is $Q(E)_{k}^{\prime}$ - 
automorphic, $\delta\left(\theta^{k}(\lambda)\right) \mid \mathcal{U}^{k}$ is transferred onto $\delta\left(\theta^{\prime k}\left(\lambda^{\prime}\right)\right) \mid \mathcal{U}^{\prime k}$. Since $E$ is $k$-reducible to $E^{\prime}$, we see that $\theta^{k}(\lambda)$ is weakly $k$-reducible to $\theta^{\prime k}\left(\lambda^{\prime}\right)$.

If $\mathcal{Q}(E)$ is $k$-closed at $\left(x_{0}, f\right)$, we see that $\mathcal{Q}\left(\theta^{k}(\lambda)\right)=\mathbb{Q}(E)$ on a neighborhood of $j_{x_{0}}^{k}(f)$. Therefore the condition (3) in Definition 6.1 is also satisfied, namely, $\theta^{k}(\lambda)$ is $k$-reducible to $\theta^{\prime k}\left(\lambda^{\prime}\right)$ by $\pi$. This completes the proof of Lemma 4.1.1.

Remark 4.1.3. For a pair $\left(E, E^{\prime}\right)$ which does not satisfy the reducibility, $D^{k}(\pi)$ is also defined if $\mathcal{Q}(E) \mid \mathcal{Q}^{k}$ is transferred by $\pi$ to $\mathscr{Q}\left(E^{\prime}\right) \mid \mathcal{U}^{\prime k}$ for a neighborhood $\mathcal{Q}^{k}$ or $\mathcal{U}^{\prime k}$.

Proposition 4.1.1. Let $\left(Q, Q^{\prime}, \pi\right)$ be a fibred manifold, and let $E$ be a differential equation at $j_{x_{0}}^{\alpha}(f) \in \tilde{J}^{\alpha}(N, Q)$ which is $K$-reducible to a differential equation $E^{\prime}$ at $j_{x_{0}}^{\alpha^{\prime}}(\pi \circ f) \in \tilde{J}^{\alpha^{\prime}}\left(N, Q^{\prime}\right)$ by $\pi$. Suppose that $E$ and $E^{\prime}$ are $l$-regular $\left(l \geqslant \alpha, \alpha^{\prime}\right)$ at $x_{0}$. We set $l^{\prime}=\max (l, K)$. Then the following two assertions hold:

(1) If $p^{l^{\prime-\alpha}}(E)$ is an $\mathcal{L}_{\mathscr{Q}(E)}$-orbit system at $\left(l^{\prime}, x_{0}, f\right)$, then $p^{l^{\prime}-\alpha^{\prime}}\left(E^{\prime}\right)$ is also an $\mathcal{L}_{\mathfrak{Q}\left(E^{\prime}\right)}$-orbit system at $\left(l^{\prime}, x_{0}, \pi \circ f\right)$.

(2) If $K=0$, and $E$ is $Q(E)$-automorphic, then $E^{\prime}$ is $Q\left(E^{\prime}\right)$-automorphic.

Proof. By Lemma 4.1.1. $D^{l^{\prime}}(\pi)$ induces a map of $\delta\left(D^{K^{l^{\prime}}}\left(\theta^{l^{\prime}}, E\right)\right) \mid \mho^{K^{l^{\prime}}}$ onto $\left.\mathcal{S}\left(D^{K^{\prime \prime}}\left(\theta^{\prime l^{\prime}}, E^{\prime}\right)\right)\right|^{\mathscr{Q}} \widetilde{K}^{K^{\prime \prime}}$. Since $p^{l^{\prime}-\alpha}(E)$ is an $\mathcal{L}_{\mathfrak{Q}(E)}$-orbit system at $\left(l^{\prime}, x_{0}, f\right), \delta\left(D^{K^{\prime}}\left(\theta^{l^{\prime}}, E\right)\right) \mid \mathcal{Q r}^{K^{\prime^{\prime}}}$ consists of a single point. Therefore $\mathcal{S}\left(D^{K^{\prime \prime}}\left(\theta^{\prime l^{\prime}}, E^{\prime}\right)\right) \mid \mathcal{Q}^{K^{\prime \prime}}$ also consists of a single point. This means that $p^{l^{\prime}-\alpha^{\prime}}\left(E^{\prime}\right)$ is also an $\mathcal{L}_{\mathbb{Q}\left(E^{\prime}\right)}$-orbit system at $\left(l^{\prime}, x_{0}, \pi \circ f\right)$. This proves (1).

Suppose $E$ is $Q(E)$-automorphic. Then any $s \in \mathcal{S}(E)$ is of the form $g \circ f$, $g \in \mathbb{Q}(E)$. Let $s^{\prime}$ be any element of $\mathcal{S}\left(E^{\prime}\right) \mid \mathcal{Q}^{\prime 0}$ where $\mathcal{U}^{\prime 0}$ is a neighborhood of $\left(x_{0}, \pi \circ f\left(x_{0}\right)\right) \in N \times Q^{\prime}$. Then by the 0-reducibility of $E$ to $E^{\prime}$ by $\pi$, there is an $\tilde{s} \in \mathcal{S}(E) \mid \mathcal{Q}^{0}$ such that $s^{\prime}=\pi \circ \tilde{s}$, and that $\mathcal{Q}^{0}$ is a neighborhood of $\left(x_{0}, f\left(x_{0}\right)\right) \in N \times Q$. As was proved in Lemma 4.1.1, for any $g \in \mathbb{Q}(E) \mid \mathcal{Q}^{0}$, we have a unique $g^{\prime} \in \mathbb{Q}\left(E^{\prime}\right) \mid \mathcal{U}^{\prime 0}$ with $g^{\prime} \circ \pi=\pi \circ g$. Therefore we get $\tilde{s}^{\prime}=\pi \circ \tilde{s}=\pi \circ g \circ f=g^{\prime} \circ \pi \circ f$. This implies that $E^{\prime}$ is $\mathcal{Q}\left(E^{\prime}\right)$-automorphic. This proves (2).

\section{Pseudo-isomorphism of automorphism pseudo-groups}

5.1. Let $\left(Q, Q^{\prime}, \pi\right)$ be a fibred manifold, and let $\Gamma$ and $\Gamma^{\prime}$ be pseudogroups on $Q$ and $Q^{\prime}$ respectively.

Definition 5.1.1. $\Gamma$ is said to be $k$-pseudo-isomorphic to $\Gamma^{\prime}$ by $\pi$ at $(q, \pi(q)) \in Q \times Q^{\prime}$ if there exist neighborhoods $\mathcal{Q}^{k}$ and $\mathcal{Q}^{\prime k}$ of $j_{q}^{k}(1) \in$ $\tilde{J}^{k}(Q, Q)$ and $j_{\pi(q)}^{k}(1) \in \tilde{J}^{k}\left(Q^{\prime}, Q^{\prime}\right)$, respectively, satisfying the following conditions:

(1) For any $g: U \rightarrow V$ which belongs to $\Gamma \mid \mathcal{Q}^{k}$, there exists a map $g^{\prime}$ : $\pi(U) \rightarrow \pi(V)$ belonging to $\Gamma^{\prime} \mid \mathcal{U}^{\prime k}$ such that $\pi \circ g=g^{\prime} \circ \pi$ on $U$. 
(2) For any $g^{\prime}: U^{\prime} \rightarrow V^{\prime}$ which belongs to $\Gamma^{\prime} \mid \mathcal{U}^{\prime k}$, there exists a map $g$ : $U \rightarrow V$ belonging to $\Gamma \mid \mathcal{U}^{k}$ such that $\left(U, U^{\prime}, \pi\right)$ is a fibred manifold and $\pi \circ g=g^{\prime} \circ \pi$ on $U$.

(3) Let $g: U \rightarrow V$ and $\tilde{g}: \tilde{U} \rightarrow \tilde{V}$ be two maps which belong to $\Gamma \mid Q^{k}$ such that $\left(U, U^{\prime}, \pi\right)$ and $\left(\tilde{U}, U^{\prime}, \pi\right)$ are fibred manifolds and such that $\pi \circ g=$ $g^{\prime} \circ \pi$ on $U$ and $\pi \circ \tilde{g}=\mathrm{g}^{\prime} \circ \pi$ on $\tilde{U}$. Then we have $g=\tilde{g}$ on $U \cap \tilde{U}$.

Remark 5.1.1. If $\Gamma$ is $k$-pseudo-isomorphic to $\Gamma^{\prime}$ by $\pi$ at $(q, \pi(q))$, there is a neighborhood $\mathcal{Q}$ of $q$ such that $\Gamma$ is $k$-pseudo-isomorphic to $\Gamma^{\prime}$ by $\pi$ at $(p, \pi(p))$ for any $p \in \mathcal{Q}$.

Lemma 5.1.1. Let $\left(Q, Q^{\prime}, \pi\right)$ be a fibred manifold, and let $\Gamma$ (resp. $\left.\Gamma^{\prime}\right)$ be a pseudo-group on $Q$ (resp. $\left.Q^{\prime}\right)$, which is complete at $(z, 1)($ resp. $(\pi(z), 1)$ ) for any $z \in Q$. Suppose $\operatorname{dim} N \geqslant \operatorname{dim} Q$, and let $f$ be a local submersion of $a$ neighborhood $\mathcal{T}$ of $x_{0}$ onto $\mathcal{U} \subset Q$. For a sufficiently large integer $l$, we assume that there are neighborhoods $\mathcal{Q}^{l}$ of $j_{x_{0}}^{l}(f) \in \tilde{J}^{l}(N, Q)$ and $\mathcal{U}^{\prime l}$ of $j_{x_{0}}^{l}(\pi \circ f) \in$ $\tilde{J}^{l}\left(N, Q^{\prime}\right)$ satisfying the following conditions:

(1) $\left(\mathcal{U}^{l}, \mathscr{U}^{\prime l}, \pi^{l}\right)$ is a fibred manifold.

(2) $\varrho_{\Gamma}(l, x, f)$ is weakly $K$-reducible to $\varrho_{\Gamma^{\prime}}(l, x, \pi \circ f)$ for any $x \in \mathcal{V}$.Then $\Gamma$ is $l^{\prime}$-pseudo-isomorphic to $\Gamma^{\prime}$ by $\pi$ at $\left(f\left(x_{0}\right), \pi \circ f\left(x_{0}\right)\right), l^{\prime}=\max (l, K)$.

Proof. We set $f^{\prime}=\pi \circ f$. For a map $g: U \rightarrow V$ belonging to $\Gamma \mid \mathcal{Q}^{l^{\prime}}, g \circ f$ is also in $\mathcal{S}\left(\mathfrak{\complement}_{T}\left(l, x_{0}, f\right), \mathcal{Q}^{l^{\prime}}\right)$. If we set $\tilde{f}=g \circ f$ and $\tilde{f}^{\prime}=\pi \circ \tilde{f}, \tilde{f}^{\prime}: f^{-1}(U) \rightarrow$ $\pi(V)$ is a solution of $\varrho_{\Gamma^{\prime}}\left(l, x_{0}, f^{\prime}\right)$ and is also a solution of $\varrho_{\Gamma^{\prime}}\left(l, x, f^{\prime}\right)$ for $x \in f^{-1}(U)$. Since $\mathcal{L}_{\Gamma^{\prime}}\left(l, x, f^{\prime}\right)$ is, by [6, Theorem 6.1], $\Gamma^{\prime}$-automorphic for a sufficiently large integer $l$, there is a $g_{x}^{\prime} \in \Gamma^{\prime} \mid \mathcal{U}^{\prime l^{\prime}}$ such that $\tilde{f}^{\prime}=g_{x}^{\prime} \circ f^{\prime}$ on a neighborhood of $x$. Since $f^{\prime}$ is a submersion, we have clearly $g_{x}^{\prime} \circ \pi=\pi \circ g$ on a neighborhood of $U_{x} \subset U$ of $f(x)$. It is obvious that if $U_{x} \cap U_{y} \neq \varnothing$, we have $g_{x}^{\prime}=g_{y}^{\prime}$ on $\pi\left(U_{x}\right) \cap \pi\left(U_{y}\right)$. This implies that there exists a map $g^{\prime}$ : $\pi(U) \rightarrow \pi(V)$ belonging to $\Gamma^{\prime} \mid \mathcal{U}^{\prime \prime^{\prime}}$ such that $\pi \circ g=g^{\prime} \circ \pi$ on $U$.

Conversely let $g^{\prime}: U^{\prime} \rightarrow V^{\prime}$ belong to $\Gamma^{\prime} \mid \mathcal{U}^{\prime l^{\prime}}$ and set $\tilde{f}^{\prime}=g^{\prime} \circ f^{\prime}$. Then $\tilde{f}^{\prime}$ is a solution of $\mathscr{E}_{T^{\prime}}\left(l, x, f^{\prime}\right)$ for any $x \in\left(f^{\prime}\right)^{-1}\left(U^{\prime}\right)$. Since $\mathfrak{E}_{\Gamma}(l, x, f)$ is weakly $K$-reducible to $\mathfrak{E}_{\Gamma^{\prime}}\left(l, x, f^{\prime}\right)$, we have an element $s_{x} \in \mathcal{S}\left(\mathfrak{E}_{\Gamma}(l, x, f)\right) \mid \mathcal{Q}^{l^{\prime}}$ such that $\tilde{f}^{\prime}=\pi \circ s_{x}$. Then since $\mathfrak{E}_{\Gamma}(l, x, f)$ is $\Gamma$-automorphic, $s_{x}$ is of the form $s_{x}=g_{x} \circ f$ on a neighborhood of $x$, where $g_{x} \in \Gamma \mid \mathcal{Q l}^{\prime \prime}$ and $g_{x}$ is defined on $U_{x}$ which satisfies $\pi\left(U_{x}\right) \subset U^{\prime}$. We set $U=\cup_{x \in\left(f^{\prime}\right)^{-1}(U)} U_{x}$. Then we can easily see that $\left(U, U^{\prime}, \pi\right)$ is a fibred manifold. Moreover if $U_{x} \cap U_{y} \neq \varnothing$, then we have $\pi \circ s_{x}=\pi \circ s_{y}$ on $f^{-1}\left(U_{x} \cap U_{y}\right)$. For $\tilde{x} \in f^{-1}\left(U_{x} \cap U_{y}\right)$, both $s_{x}$ and $s_{y}$ are elements of $\delta\left(\complement_{\Gamma}(l, \tilde{x}, f)\right) \mid \mathcal{Q}^{l^{\prime}}$. Therefore, by the reducibility, $s_{x}=s_{y}$ on a neighborhood of $\tilde{x}$. Thus we get $s_{x}=s_{y}$ on $f^{-1}\left(U_{x} \cap U_{y}\right)$, that is, $g_{x} \circ f=$ $g_{y} \circ f$ on $f^{-1}\left(U_{x} \cap U_{y}\right)$. Since $f$ is a submersion, we get $g_{x}=g_{y}$ on $U_{x} \cap U_{y}$. This implies that we have an element $g \in \Gamma \mid \mathcal{U l}^{l^{\prime}}$ defined on $U$ such that $\pi \circ g=g^{\prime} \circ \pi$. 
Let $\tilde{g}: \tilde{U} \rightarrow \tilde{V}$ be another element of $\Gamma \mid \mathcal{U}^{l^{\prime}}$ such that $g^{\prime} \circ \pi=\pi \circ \tilde{g}$ and $\left(\tilde{U}, U^{\prime}, \pi\right)$ is a fibred manifold. Then for any $x \in f^{-1}(U \cap \tilde{U}), g \circ f$ and $\tilde{g} \circ f$ are elements of $\mathcal{S}\left(\complement_{T}(l, x, f)\right) \mid \mathcal{U}^{l^{\prime}}$. Since $\pi \circ g \circ f=\pi \circ \tilde{g} \circ f \in$ $\delta\left(\mathcal{L}_{\Gamma^{\prime}}\left(l, x, f^{\prime}\right)\right) \mid \mathcal{Q}^{\prime \prime^{\prime}}$, we get $g \circ f=\tilde{g} \circ f$ on a neighborhood of $x$. Since $x$ is an arbitrary point of $f^{-1}(U \cap \tilde{U})$, we have $g \circ f=\tilde{g} \circ f$ on $f^{-1}(U \cap \tilde{U})$. Since $f$ is a submersion, we obtain $=\tilde{g}$ on $U \cap \tilde{U}$. This proves that $\Gamma$ is $l^{\prime}$-pseudo-isomorphic to $\Gamma^{\prime}$ at $\left(f\left(x_{0}\right), \pi \circ f\left(x_{0}\right)\right)$.

Lemma 5.1.2. Suppose $\operatorname{dim} N \geqslant \operatorname{dim} Q$. Let $\Gamma$ be a pseudo-group on $Q$ which is complete at $\left(z_{0}, 1\right)$, and let $f$ be a submersion of a neighborhood of $x_{0} \in N$ to $Q$ with $f\left(x_{0}\right)=z_{0}$. We denote by $\tilde{l}$ the order of $\mathscr{E}_{\Gamma}$ at $\left(x_{0}, f\right)$. Then, for $l \geqslant \tilde{l}$, there is a neighborhood $\mathcal{Q}^{l}$ of $j_{x_{0}}^{l}(f)$ such that $\mathcal{Q}\left(\varrho_{T}\left(\tilde{l}, x_{0}, f\right)\right) \mid \mathcal{U}^{l}=$ $\Gamma \mid \mathcal{Q}^{l}$.

Proof. Let $\left\{\boldsymbol{\theta}_{j}^{\tilde{l}}\right\}_{j=1}^{m_{i}}$ be a fundamental system of differential invariants of $\mathcal{L}_{\Gamma}$ at $j_{x_{0}}^{\tilde{l}}(f)$. We assume that each $\theta_{j}^{\tilde{l}}$ is defined on a neighborhood $\mathfrak{\mathcal { i }}$ of $j_{x_{0}}^{\tilde{l}}(f)$, and $\beta^{\tilde{l}}(\mathfrak{V}) \supset \operatorname{Im} f$. We set $\mathscr{U}=\operatorname{Im} f$. Then $\varrho_{\Gamma}(\tilde{l}, x, f)$ is defined for any $x \in \mathcal{Q}$. Let $\phi: U\left(\exists z_{0}\right) \rightarrow V\left(\exists z_{0}\right)$ belong to $\mathbb{Q}\left(\mathfrak{E}_{T}\left(\tilde{l}, x_{0}, f\right)\right) \mid थ$. Then $\tilde{f}=$ $\phi \circ f$ is a solution of $\mathcal{L}_{\Gamma}(\tilde{l}, x, f)$ for any $x \in f^{-1}(U)$. Since by [6, Theorem 6.1], $\varrho_{\Gamma}(\tilde{l}, x, f)$ is $\Gamma$-automorphic, we have a $g_{x} \in \Gamma$ such that $f=g_{x} \circ f$ on a neighborhood of $x$. Since $f$ is a submersion, we see that $\phi=g_{x}$ on a neighborhood $U_{x} \subset U$ of $f(x)$. Moreover we have clearly $g_{x}=g_{y}$ on $U_{x} \cap$ $U_{y}$. This implies that $\phi \in \Gamma \mid \mathcal{Q}$ and any restriction of $\phi$ to an open subset of $U$ belongs to $\Gamma \mid \mathcal{U}$. This means that $\mathcal{Q}\left(\mathfrak{E}_{\Gamma}\left(\tilde{l}, x_{0}, f\right)\right)|\mathcal{U} \subset \Gamma| \mathcal{U}$. Let $\mathcal{U}^{l}, l>\tilde{l}$, be a neighborhood of $j_{x_{0}}^{l}(f)$ such that $\left(\mathcal{Q l}^{l}, \mathcal{Q}, \pi^{l}\right)$ is a fibred manifold. Then we have $\mathbb{Q}\left(\mathfrak{E}_{\Gamma}\left(\tilde{l}, x_{0}, f\right)\right)\left|\mathcal{Q}^{l} \subset \Gamma\right| \mathcal{Q}^{l}$. Conversely we have clearly $\Gamma \mid \mathcal{Q}^{l} \subset$ $\mathcal{Q}\left(\varrho_{T}\left(\tilde{l}, x_{0}, f\right)\right) \mid \mathcal{U}^{l}$. This completes the proof of Lemma 7.2.

5.2. Theorem 5.2.1. Let $\left(Q, Q^{\prime}, \pi\right)$ be a fibred manifold, and let $\mathcal{L}$ (resp. $\left.\mathcal{L}^{\prime}\right)$ be a regular weak Lie algebra sheaf on $Q\left(\right.$ resp. $\left.Q^{\prime}\right)$. Assume $\operatorname{dim} N>\operatorname{dim} Q$. If $\mathcal{L}\left(l, x_{0}, f\right)$ is weakly $K$-reducible to $\mathcal{L}^{\prime}\left(l, x_{0}, \pi \circ f\right)$ by $\pi$ for a sufficiently large integer $l$, then $\mathbb{Q}\left(\mathcal{L}\left(l, x_{0}, f\right)\right)$ is $k$-pseudo-isomorphic to $\mathcal{Q}\left(\mathcal{L}^{\prime}\left(l, x_{0}, \pi \circ f\right)\right)$ by $\pi$ at $\left(f\left(x_{0}\right), \pi \circ f\left(x_{0}\right)\right)$ for $k \geqslant l^{\prime}=\max (l, K)$.

Conversely, if $\mathbb{Q}\left(\mathcal{L}\left(l, x_{0}, f\right)\right)$ is $k$-pseudo-isomorphic to $\mathbb{Q}\left(\mathcal{L}^{\prime}\left(l, x_{0}, \pi \circ f\right)\right)$ for a sufficiently large $l$ and an integer $k \geqslant l$, then $\mathcal{L}\left(l, x_{0}, f\right)$ is $k$-reducible to $\mathcal{L}^{\prime}\left(l, x_{0}, \pi \circ f\right)$ by $\pi$.

Proof. Let $\Gamma$ or $\Gamma^{\prime}$ be a pseudo-group on $Q$ or $Q^{\prime}$ such that $\mathscr{E}_{\Gamma}=\mathcal{E}$ or $\mathfrak{E}_{\Gamma^{\prime}}=\mathcal{L}^{\prime}$ and such that $\Gamma$ or $\Gamma^{\prime}$ is complete at $(z, 1)$ or $(\pi(z), 1)$ for any $z \in Q$. By Lemma 5.1.2 we have $\mathcal{Q}\left(\mathfrak{L}_{\Gamma}\left(l, x_{0}, f\right)\right)\left|\mathcal{Q}^{l^{\prime}}=\Gamma\right| \mathcal{Q}^{l^{\prime}}$ and $\mathcal{Q}\left(\varrho_{\Gamma^{\prime}}\left(l, x_{0}, \pi \circ f\right)\right)\left|\mathcal{Q}^{\prime l^{\prime}}=\Gamma^{\prime}\right| \mathcal{U}^{\prime l^{\prime}}$ for some neighborhoods $\mathcal{U}^{l^{\prime}}$ and $\mathcal{U}^{\prime l^{\prime}}$ of $j_{x_{0}}^{l^{\prime}}(f)$ and $j_{x_{0}}^{l^{\prime}}(\pi \circ f)$, respectively.

On the other hand, it is easy to see that there is a neighborhood $\mathcal{V}$ of $x_{0}$ such that, for any $x \in \mathcal{V}, \mathcal{L}(l, x, f)$ is weakly $K$-reducible to $\mathcal{L}^{\prime}(l, x, \pi \circ f)$ by 
$\pi$. Therefore by Lemma 5.1.1, the assertion of the former half is obtained. Since $\mathcal{L}\left(l, x_{0}, f\right)$ or $\mathcal{L}^{\prime}\left(l, x_{0}, \pi \circ f\right)$ is $\mathcal{Q}\left(\mathcal{L}\left(l, x_{0}, f\right)\right)$-automorphic or $\mathcal{Q}\left(\mathcal{L}^{\prime}\left(l, x_{0}, \pi \circ f\right)\right)$-autormorphic, the latter half is now clear, and the proof is completed.

Theorem 5.2.2. Let $\left(Q, Q^{\prime}, \pi\right)$ be a fibred manifold, and let $E$ (resp. $\left.E^{\prime}\right)$ be a differential equation at $j_{x_{0}}^{\alpha}(f) \in \tilde{J}^{\alpha}(N, Q)$ (resp. $J_{x_{0}}^{\alpha^{\prime}}(\pi \circ f) \in \tilde{J}^{\alpha^{\prime}}\left(N, Q^{\prime}\right)$ ). Assume that $\operatorname{dim} N \geqslant \operatorname{dim} Q$, that $E$ and $E^{\prime}$ are l-regular at $x_{0}$ and that $Q(E)$ or $\mathbb{Q}\left(E^{\prime}\right)$ is complete at $\left(f\left(x_{0}\right), 1\right)$ or $\left(\pi \circ f\left(x_{0}\right), 1\right)$. If the following conditions (i) and (ii) are satisfied, then $\mathbb{Q}(E)$ is k-pseudo-isomorphic to $\mathscr{Q}\left(E^{\prime}\right)$ by $\pi$ at $\left(f\left(x_{0}\right), \pi \circ f\left(x_{0}\right)\right)$, and $D^{\tilde{K}}\left(\theta^{k}, E\right)$ is weakly $\tilde{K}$-reducible to $D^{\tilde{K}}\left(\theta^{\prime k}, E^{\prime}\right)$ by $D^{k}(\pi)$ for an integer $\tilde{K}$ :

(i) $E$ is $K$-reducible to $E^{\prime}$ by $\pi$.

(ii) For a sufficiently large integer $k, \operatorname{dim}\left(\pi^{k}\right)_{*} D_{E}^{k}=\operatorname{dim} D_{E^{\prime}}^{k}$ and $\mathbb{Q}(E)_{k}^{\prime}$ is complete at $\left(\pi \circ f\left(x_{0}\right), 1\right)$.

Conversely, for a sufficiently large integer $k$, if $\mathbb{C}(E)$ is $k$-pseudo-isomorphic to $\mathcal{Q}\left(E^{\prime}\right)$ by $\pi$ at $\left(f\left(x_{0}\right), \pi \circ f\left(x_{0}\right)\right)$, and $D^{K}\left(\theta^{k}, E\right)$ is weakly $K$-reducible to $D^{K}\left(\theta^{\prime k}, E^{\prime}\right)$ by $D^{k}(\pi)$, then $E$ is $(k+K)$-reducible to $E^{\prime}$ by $\pi$, and $\operatorname{dim}\left(\pi^{k}\right)_{*} D_{E}^{k}=\operatorname{dim} D_{E^{\prime}}^{k}$

Proof. By Lemma 4.1.1 and Remark 4.1.1, for a sufficiently large $k>$ $\max (l, K), 0_{E}^{k}$ is one-to-one, and $\mathcal{L}_{\Theta(E)}\left(k, x_{0}, f\right)$ is weakly $k$-reducible to $\mathfrak{E}_{\mathfrak{Q}(E)}\left(k, x_{0}, \pi \circ f\right)$. Therefore by Theorem 5.2.1, the assertion of the former half follows. By Remark 5.1.1 and the fact that $\mathcal{L}_{Q(E)}(k, x, f)$ or $\mathcal{L}_{\mathscr{Q}(E)}(k, x, \pi \circ f)$ is $\mathscr{Q}(E)$ - or $\mathcal{Q}\left(E^{\prime}\right)$-automorphic, the latter half easily follows, and the proof is completed.

Now we shall modify the condition (i) in Theorem 5.2.2.

Let $E$ be a differential equation at $j_{x}^{\alpha}(f)$ which is $l$-regular at $x$, and let $\left\{\theta_{j}^{l}\right\}_{j=1}^{m_{l}}$ be a fundamental system of differential invariants of $\mathcal{L}_{\mathscr{Q}(E)}$ at $j_{x}^{l}(f)$. For a subset $\mathscr{F} \subset \mathcal{S}(E)$, we set $\mathcal{O}_{E}^{\mathscr{F}}=\left\{\lambda:{ }^{\exists} \in \mathscr{F}\right.$ such that $\left.\theta^{l}\left(j^{l}(s)\right)=\lambda\right\}$.

Definition 5.2.1. A differential equation $E$ is said to be $\mathscr{F}$-trivial if, for any $\lambda, \mu \in \mathcal{O}_{E}^{\mathscr{F}}$, there exists an element $\Delta \in D\left(\theta^{l}, \mathcal{N}(\mathscr{Q}(E))\right)$ such that $\mu=\Delta \circ \lambda$.

Let $\left(Q, Q^{\prime}, \pi\right)$ be a fibred manifold, and let $E$ and $E^{\prime}$ be differential equations at $j_{x}^{\alpha}(f)$ and $j_{x}^{\alpha^{\prime}}(\pi \circ f)$ respectively. Suppose $E$ is weakly $K$-reducible to $E^{\prime}$ by $\pi$ and $f \in \mathcal{S}(E)$. We set $\tilde{\mathcal{F}}=\{\pi \circ s ; s$ is of the form $\phi \circ f, \phi \in$ $\mathcal{Q}(E)\}$. Then for a neighborhood $\mathcal{Q}^{\prime K}$ of $j_{x}^{K}(\pi \circ f), \mathcal{F}=\tilde{\mathscr{F}} \mid \mathcal{Q}^{K}$ is a subset of $\delta\left(E^{\prime}\right)$.

Theorem 5.2.3. The condition (i) in Theorem 5.2.2 is equivalent to the following condition $\left(\mathrm{i}^{\prime}\right)$ :

(i') $E$ is weakly $K$-reducible to $E^{\prime}$ by $\pi$, and $E^{\prime}$ is $\mathscr{F}$-trivial.

Proof. Let $\phi \in \mathbb{Q}(E) \mid \mathcal{Q}^{K}$ and set $g=\phi \circ f$. Then $g \in \mathcal{S}(E) \mid \mathcal{Q}^{K}$. Since $E^{\prime}$ is $\mathscr{F}$-trivial, $\mathcal{L}_{\mathbb{Q}\left(E^{\prime}\right)}(l, x, \pi \circ f)$ is isomorphic to $\mathcal{L}_{\mathbb{Q}(E)}(l, x, \pi \circ g)$ by Proposi- 
tion 3.1.1. Let $\varphi$ be such an isomorphism. We set $f^{\prime}=\pi \circ f$ and $\varphi \circ f^{\prime}=g^{\prime \prime}$. Then $g^{\prime \prime} \in \mathcal{S}\left(\mathfrak{L}_{\mathscr{Q}\left(E^{\prime}\right)}(l, x, \pi \circ g)\right)$. Since $\mathfrak{L}_{\mathscr{Q}(E)}(l, x, \pi \circ g)$ is $\mathcal{Q}\left(E^{\prime}\right)$-automorphic, we have an $\xi \in \mathbb{Q}\left(E^{\prime}\right)$ such that $\xi \circ g^{\prime \prime}=\pi \circ g$. We set $\phi^{\prime}=\xi \circ \varphi$. Then we have $\pi \circ \phi \circ f=\phi^{\prime} \circ \pi \circ f$. Since $f$ is a submersion, we get $\pi \circ \phi=$ $\phi^{\prime} \circ \pi$. Sinc $\left(E, E^{\prime}\right)$ is a weakly $K$-reduced pair, we see that $\phi^{\prime} \in \mathbb{Q}\left(E^{\prime}\right)$. That is, $E$ is $K$-reducible to $E^{\prime}$ by $\pi$. Conversely, if we assume (i), it is easy to see that the condition ( $\left.i^{\prime}\right)$ is satisfied. Hence the proof is completed.

5.3. Example 1. We set $\mathbf{R}_{*}^{2}=\left\{\left(z_{1}, z_{2}\right) \in \mathbf{R}^{2} \mid z_{2} \neq 0\right\}$, and let $E$ be the differential equaton at $j_{x_{0}}^{1}(f) \in \tilde{J}^{1}\left(\mathbf{R}^{2}, \mathbf{R}_{*}^{2}\right)$ generated by $z_{2} \cdot \partial z_{1} / \partial x_{1}-$ $\alpha\left(x_{1}, x_{2}\right)$ and $z_{2} \cdot \partial z_{1} / \partial x_{2}-\beta\left(x_{1}, x_{2}\right)$, where $\left\{x_{1}, x_{2}\right\}$ is the coordinate system on $N=\mathbf{R}^{2}$, and $\alpha(x)=\left[z_{2} \cdot \partial z_{1} / \partial x_{1}\right]\left(j_{x}^{1}(f)\right), \beta(x)=\left[z_{2} \cdot \partial z_{1} / \partial x_{2}\right]\left(j_{x}^{1}(f)\right)$.

On the other hand, we assume $\alpha(x) \neq 0$ and denote by $E^{\prime}$ the differential equation at $j_{x_{0}}^{1}\left(f^{\prime}\right) \in \tilde{J}^{1}\left(\mathbf{R}^{2}, \mathbf{R}\right)$ generated by $\left(\partial z_{1} / \partial x_{2}\right) /\left(\partial z_{1} / \partial x_{1}\right)-\beta / \alpha$, where $f^{\prime}=\pi \circ f$, and $\pi$ is the projection of $\mathbf{R}_{*}^{2}$ onto $\mathbf{R}$ defined by $z_{1}=$ $\pi\left(z_{1}, z_{2}\right)$.

We shall show the following two assertions:

(1) $E$ is 1-reducible to $E^{\prime}$ by $\pi$.

(2) $\mathscr{Q}(E)$ is 1-pseudo-isomorphic to $\mathbb{Q}\left(E^{\prime}\right)$ by $\pi$ at $\left(f\left(x_{0}\right), f^{\prime}\left(x_{0}\right)\right)$.

Let $\mathcal{L}$ be the sheaf of germs of local vector fields on $\mathbf{R}_{* 2}$ of the form $\xi\left(z_{1}\right) \cdot \partial / \partial z_{1}-\xi^{\prime}\left(z_{1}\right) \cdot z_{2} \cdot \partial / \partial z_{2}$, where $\xi$ is any local function on $\mathbf{R}$. ( $\mathfrak{L}$ is given in [4] and [7]). Then $\mathcal{L}$ is a regular Lie algebra sheaf on $\mathbf{R}_{*}^{2}$, and we can easily see that the family $\left\{x_{1}, x_{2}, z_{2} \cdot \partial z_{1} / \partial x_{1}, z_{2}\right.$. $\left.\partial z_{1} / \partial x_{2}, D\left(z_{1}, z_{2}\right) / D\left(x_{1}, x_{2}\right)\right\}$ is a fundamental system of differential invariants of $\mathcal{L}$ at $j_{x}^{1}(f)$, and the order of $\mathcal{L}$ at $\left(x_{0}, f\right)$ is 1 . Therefore, if we denote by $\tilde{E}$ the differential equation at $j_{x}^{1}(f)$ generated by $z_{2} \cdot \partial z_{1} / \partial x_{1}-\alpha$, $z_{2} \cdot \partial z_{1} / \partial x_{2}-\beta$ and $D\left(z_{1}, z_{2}\right) / D\left(x_{1}, x_{2}\right)-\left(\partial \alpha / \partial x_{2}-\partial \beta / \partial x_{1}\right)$, then $\tilde{E}$ possesses a solution, and $\mathscr{Q}(\tilde{E})=\Gamma$ on a neighborhood $\mathcal{U}^{1}$ of $j_{x_{0}}^{1}(f)$, where $\Gamma$ is a pseudo-group given on a neighborhood of $f\left(x_{0}\right)$ such that $\Gamma$ is complete at $\left(f\left(x_{0}\right), 1\right)$ and $\mathfrak{e}_{\mathrm{T}}=\mathfrak{L}$.

On the other hand, it is easy to see that we have $\delta(\tilde{E})=\delta(E)$. Therefore we get $\mathscr{Q}(E)=\mathscr{Q}(\tilde{E})$.

Now let $\mathfrak{L}^{\prime}$ be the sheaf of germs of all local vector fields on $\mathbf{R}$. Then clearly $\mathcal{L}^{\prime}$ is a regular Lie algebra sheaf on $\mathbf{R}$, and $\left\{x_{1}, x_{2},\left(\partial z_{1} / \partial x_{2}\right) /\left(\partial z_{1} / \partial x_{1}\right)\right\}$ is a fundamental system of differential invariants of $\mathcal{L}^{\prime}$ at $j_{x}^{1}\left(f^{\prime}\right) \in \tilde{J}^{1}\left(\mathbf{R}^{2}, \mathbf{R}\right)$. Therefore $\mathscr{Q}\left(E^{\prime}\right)$ is the pseudo-group on a neighborhood $\mathcal{U}^{\prime}$ of $f^{\prime}\left(x_{0}\right)$ consisting of all local diffeomorphism of $\mathcal{U}^{\prime}$. We set $\mathcal{Q}=\pi^{-1}\left(\mathcal{U}^{\prime}\right),\left(\beta^{k}\right)^{-1}(\mathcal{Q})=\mathcal{Q}^{k},\left(\beta^{k}\right)^{-1}\left(\mathcal{U}^{\prime}\right)=\mathcal{Q}^{\prime k}$ for $k \geqslant 1$. Then $\left(Q^{k}, Q^{\prime k}, \pi^{k}\right)$ is a fibred manifold $(k \geqslant 1)$. Let $s \in \delta(E) \mid Q^{k}$. Then it is clear that $s^{\prime}=\pi \circ s \in \mathcal{S}\left(E^{\prime}\right) \mid \mathcal{Q}^{\prime k}$. Conversely, let $s^{\prime} \in \mathcal{S}\left(E^{\prime}\right) \mid Q^{\prime k}$. Then $\left(\partial s^{\prime} / \partial x_{2}\right) /\left(\partial s^{\prime} / \partial x_{1}\right)=\beta / \alpha$. Therefore $\alpha /\left(\partial s^{\prime} / \partial x_{1}\right)=\beta /\left(\partial s^{\prime} / \partial x_{2}\right)$. We shall 
define a local map $s$ of $\mathbf{R}^{2}$ to $\mathbf{R}_{*}^{2}$ by $z_{1}(s)=s^{\prime}$ and $z_{2}(s)=\alpha /\left(\partial s^{\prime} / \partial x_{1}\right)$. Then, since $\left(D\left(z_{1}(f), z_{2}(f)\right) / D\left(x_{1}, x_{2}\right)\right)_{x=x_{0}} \neq 0$, there exists a neighborhood $\mathcal{V}^{\prime k} \subset$ $\mathcal{Q}^{\prime k}$ of $j_{x}^{k}\left(f^{\prime}\right)$ such that, for any $\left.s^{\prime} \in \mathcal{S}\left(E^{\prime}\right)\right|^{\mathfrak{V}^{\prime k}}$, if we construct $s$ from $s^{\prime}$ by the above stated way, we have $\left(D\left(z_{1}(s), z_{2}(s)\right) / D\left(x_{1}, x_{2}\right)\right)_{x=x_{0}} \neq 0$. We set $\left(\pi^{k}\right)^{-1}\left(\mathcal{V}^{\prime k}\right)=\mathcal{V}^{k}$. Then $\left(\mathcal{V}^{k}, \mathcal{V}^{\prime k}, \pi^{k}\right)$ is a fibred manifold, and $\left.\mathcal{S}(E)\right|^{\mathcal{T}^{k}}$ and $\left.\delta\left(E^{\prime}\right)\right|^{\mathcal{V}^{\prime k}}$ satisfy the condition (2) of Definition 4.1.1 for $k \geqslant 1$. This proves that $\tilde{E}$ and therefore $E$ are weakly 1-reducible to $E^{\prime}$ by $\pi$. By Theorem 5.2.1, $\mathcal{Q}(\tilde{E})$ and therefore $\mathscr{Q}(E)$ are 1-pseudo-isomorphic to $\mathcal{Q}\left(E^{\prime}\right)$ by $\pi$ at $\left(f\left(x_{0}\right), f^{\prime}\left(x_{0}\right)\right)$.

Example 2. Let $E$ denote the system of differential equation at $j_{x_{0}}^{1}(f) \in$ $\tilde{J}^{1}\left(\mathbf{R}^{2}, \mathbf{R}_{*}^{2}\right)$ given by

$$
\frac{\partial z_{1}}{\partial x_{1}}=z_{2}, \quad \frac{\partial z_{1}}{\partial x_{2}}=0,
$$

and let $E^{\prime}$ be the differential equation at $j_{x_{0}}^{2}\left(f^{\prime}\right) \in \tilde{J}^{2}\left(\mathbf{R}^{2}, \mathbf{R}\right)$ given by

$$
\frac{\partial^{2} z_{1}}{\partial x_{1} \partial x_{2}}=0
$$

where $f^{\prime}=\pi \circ f$, and $\pi$ is the projection of $\mathbf{R}_{*}^{2}$ to $\mathbf{R}$ defined by $z_{1}=\pi\left(z_{1}, z_{2}\right)$. We shall prove that $E$ is 1-reducible to $E^{\prime}$ by $\pi$, and that $Q(E)$ is 0-pseudoisomorphic to $\mathcal{Q}\left(E^{\prime}\right)$ by $\pi$ at $\left(f\left(x_{0}\right), f^{\prime}\left(x_{0}\right)\right)$.

It is clear that $E$ is weakly 1-reducible to $E^{\prime}$ by $\pi$.

Now we shall calculate the automorphism pseudo-groups $\mathscr{Q}(E)$ and $\mathbb{Q}\left(E^{\prime}\right)$. Let $\phi \in \mathbb{Q}\left(E^{\prime}\right)$, and set $\overline{s^{\prime}}=\phi \circ s^{\prime}$. Then, if $\partial^{2} s^{\prime} / \partial x_{1} \partial x_{2}=0$, we have $\partial^{2} \overline{s^{\prime}} / \partial x_{1} \partial x_{2}=0$. Since

$$
\frac{\partial^{2} \overline{s^{\prime}}}{\partial x_{1} \partial x_{2}}=\frac{\partial^{2} \phi}{\partial z_{1}^{2}} \cdot \frac{\partial s^{\prime}}{\partial x_{1}} \cdot \frac{\partial s^{\prime}}{\partial x_{2}}+\frac{\partial \phi}{\partial z_{1}} \frac{\partial^{2} s^{\prime}}{\partial x_{1} \partial x_{2}}
$$

and $\partial^{2} s^{\prime} / \partial x_{1} \partial x_{2}=0$, we get $\partial^{2} \phi / \partial z_{1}^{2} \cdot \partial s^{\prime} / \partial x_{1} \cdot \partial s^{\prime} / \partial x_{2}=0$ for any $s^{\prime} \in$ $\mathcal{S}\left(E^{\prime}\right)$. For any $\left(z_{0}, p_{0}, q_{0}\right) \in \mathbf{R}^{3}$, we have an $s^{\prime} \in \mathcal{S}\left(E^{\prime}\right)$ such that $s\left(x_{0}\right)=z_{0}$, $\left(\partial s^{\prime} / \partial x_{1}\right)\left(x_{0}\right)=p_{0}$ and $\left(\partial s^{\prime} / \partial x_{2}\right)\left(x_{0}\right)=q_{0}$. Therefore we get $\partial^{2} \phi / \partial z_{1}^{2}=0$. This implies that $\phi\left(z_{1}\right)=a \cdot z_{1}+b$, where $a$ and $b$ are constants such that $a \neq 0$. Conversely, let $\phi$ be a local diffeomorphism of $\mathbf{R}$ such that $\phi\left(z_{1}\right)=a$. $z_{1}+b$, where $a$ and $b$ are constants such that $a \neq 0$. Then it is clear that $\phi \in \mathbb{Q}\left(E^{\prime}\right)$.

Next let $\psi \in \mathbb{Q}(E)$ and $s \in \mathcal{S}(E)$, and set $\overline{z_{1}}=z_{1} \circ \psi$ and $\overline{z_{2}}=z_{2} \circ \psi$. Then we have

$$
\begin{array}{cc}
\frac{\partial z_{1}(s)}{\partial x_{1}}=z_{2}(s), & \frac{\partial z_{2}(s)}{\partial x_{2}}=0 \\
\frac{\partial z_{1}(\psi \circ s)}{\partial x_{1}}=z_{2}(\psi \circ s), & \frac{\partial z_{2}(\psi \circ s)}{\partial x_{2}}=0 .
\end{array}
$$


Since

$$
\begin{aligned}
\frac{\partial z_{2}(\psi \circ s)}{\partial x_{2}}= & \frac{\partial}{\partial x_{2}}\left(\frac{\partial z_{1}(\Psi \circ s)}{\partial x_{1}}\right) \\
= & \frac{\partial^{2} \bar{z}_{1}}{\partial z_{1}^{2}} \cdot \frac{\partial z_{1}(s)}{\partial x_{1}} \cdot \frac{\partial z_{1}(s)}{\partial x_{2}}+\frac{\partial^{2} \bar{z}_{1}}{\partial z_{1} \partial z_{2}} \cdot \frac{\partial z_{1}(s)}{\partial x_{1}} \cdot \frac{\partial z_{2}(s)}{\partial x_{2}} \\
& +\frac{\partial \bar{z}_{1}}{\partial z_{1}} \cdot \frac{\partial^{2} z_{1}(s)}{\partial x_{1} \partial x_{2}}+\frac{\partial^{2} \bar{z}_{1}}{\partial z_{1} \partial z_{2}} \cdot \frac{\partial z_{2}(s)}{\partial x_{1}} \cdot \frac{\partial z_{1}(s)}{\partial x_{2}} \\
& +\frac{\partial^{2} \bar{z}_{1}}{\partial z_{2}^{2}} \cdot \frac{\partial z_{2}(s)}{\partial x_{1}} \cdot \frac{\partial z_{2}(s)}{\partial x_{2}}+\frac{\partial \bar{z}_{1}}{\partial z_{2}} \cdot \frac{\partial^{2} z_{2}(s)}{\partial x_{1} \partial x_{2}}
\end{aligned}
$$

we get

$$
\frac{\partial^{2} \bar{z}_{1}}{\partial z_{1}^{2}} \cdot \frac{\partial z_{1}(s)}{\partial x_{1}} \cdot \frac{\partial z_{1}(s)}{\partial x_{2}}+\frac{\partial^{2} \bar{z}_{1}}{\partial z_{1} \partial z_{2}} \cdot \frac{\partial z_{2}(s)}{\partial x_{1}} \cdot \frac{\partial z_{1}(s)}{\partial x_{2}}=0 .
$$

Since for any $\left(z_{1}^{0}, z_{2}^{0}, p_{1}^{0}, p_{2}^{0}, q^{9}\right) \in \mathbf{R}^{5}$ with $z_{2}^{0}=p_{1}^{0} \neq 0$ there is an $s \in \delta(E)$ such that

$$
s\left(x_{0}\right)=\left(z_{1}^{0}, z_{2}^{0}\right), \quad \frac{\partial z_{1}(s)}{\partial x_{1}}\left(x_{0}\right)=p_{1}^{0}, \quad \frac{\partial z_{1}(s)}{\partial x_{2}}\left(x_{0}\right)=p_{2}^{0}
$$

and $\left(\partial z_{2}(s) / \partial x_{1}\right)\left(x_{0}\right)=q^{0}$, we get $\partial^{2} \bar{z}_{1} / \partial z_{1}^{2}=0$ and $\partial^{2} \bar{z}_{1} / \partial z_{1} \partial z_{2}=0$, which implies that $\bar{z}_{1}\left(z_{1}, z_{2}\right)=h\left(z_{2}\right) z_{1}+k\left(z_{2}\right)$ and $h\left(z_{2}\right)=$ constant $a \neq 0$.

On the other hand, we have

$$
\frac{\partial \bar{z}_{2}(s)}{\partial x_{2}}=\frac{\partial \bar{z}_{2}}{\partial z_{1}} \cdot \frac{\partial \bar{z}_{1}(s)}{\partial x_{2}}+\frac{\partial \bar{z}_{2}}{\partial z_{2}} \cdot \frac{\partial z_{2}(s)}{\partial x_{2}}
$$

so that $\left(\partial \bar{z}_{2} / \partial z_{1}\right) \partial z_{1}(s) / \partial x_{2}=0$. Since for any $\left(z_{1}^{0}, z_{2}^{0}, p_{2}^{0}\right) \in \mathbf{R}^{3}$ there is an $s \in \mathcal{S}(E)$ such that $s\left(x_{0}\right)=\left(z_{1}^{0}, z_{2}^{0}\right)$ and $\left(\partial z_{1}(s) / \partial x_{2}\right)\left(x_{0}\right)=p_{2}^{0}$, we have $\partial \bar{z}_{2} / \partial z_{1}=0$ which implies that $\bar{z}_{2}\left(z_{1}, z_{2}\right)$ is of the form $\eta\left(z_{2}\right)$. Since $\bar{z}_{1}\left(z_{1}, z_{2}\right)$ $=a \cdot z_{1}+k\left(z_{2}\right)$, we get

$$
\frac{\partial \bar{z}_{1}}{\partial x_{1}}=a \cdot \frac{\partial z_{1}}{\partial x_{1}}+k^{\prime}\left(z_{2}\right) \frac{\partial z_{2}}{\partial x_{1}} .
$$

Since $z_{2}(s)=\left(\partial z_{1} / \partial x_{1}\right)(s)$ and $\bar{z}_{2}(s)=\left(\partial \bar{z}_{1} / \partial x_{1}\right)(s)$ for any $s \in \mathcal{S}(E)$, we have $\eta\left(z_{2}(s)\right)=a \cdot z_{2}(s)+k^{\prime}\left(z_{2}(s)\right) \cdot \partial z_{2}(s) / \partial x_{1}$ for $s \in \mathcal{S}(E)$. Now for any $\left(q^{0}, z_{2}^{0}\right) \in \mathbf{R}^{2}$ there is an $s \in \mathcal{S}(E)$ such that $z_{2}(s)\left(x_{0}\right)=z_{2}^{0}$ and $\left(\partial z_{2}(s) / \partial x_{1}\right)\left(x_{0}\right)=q^{0}$. Therefore $k^{\prime}\left(z_{2}\right)=0$, that is, $k$ is also constant $(=b)$. Thus $\bar{z}_{1}\left(z_{1}, z_{z}\right)=a \cdot z_{1}+b$. Since $\bar{z}_{2}(s)=\left(\partial \bar{z}_{1} / \partial x_{1}\right)(s)=a \cdot z_{2}(s)$ for $s \in$ $\mathcal{S}(E)$ and for any $z^{0} \in \mathbf{R}$ there is an $s \in \mathcal{S}(E)$ with $s\left(x_{0}\right)=z^{0}$, we have $\bar{z}_{2}=a \cdot z_{2}$. This proves that for any $\psi \in \mathbb{Q}(E)$ we have constants $a$ and $b$ 
such that $a \neq 0, \bar{z}_{1}\left(z_{1}, z_{2}\right)=a \cdot z_{1}+b$ and $\bar{z}_{2}\left(z_{1}, z_{2}\right)=a \cdot z_{2}$. Conversely, let $\psi$ be a local diffeomorphism of $\mathbf{R}^{2}$ such that $z_{1} \circ \psi=a \cdot z_{1}+b$ and $z_{2} \circ \psi=$ $a \cdot z_{2}$, where $a$ and $b$ are constants and $a \neq 0$. Then it is obvious that $\psi \in \mathbb{Q}(E)$.

It is now clear that $\mathscr{Q}(E)$ is 0 -pseudo-isomorphic to $\mathcal{Q}\left(E^{\prime}\right)$ by $\pi$ at any $\left(p, p^{\prime}\right) \in \mathbf{R}^{2} \times \mathbf{R}$ with $p^{\prime}=\pi(p)$. Therefore $E$ is 1-reducible to $E^{\prime}$ by $\pi$.

\section{Compatibility of reduction and equivalence}

6.1. In this section we shall prove the compatibility of reducibility and equivalence of differential equations.

Let $E$ be a differential equation at $j_{x}^{\alpha}(f) \in \tilde{J}^{\alpha}(N, Q)$, and let $\delta(E)$ denote the space of solutions of $E$. Let $\mathcal{Q}^{k}$ be a neighborhood of $j_{x}^{k}(f)$, and set $\mathcal{S}(E) \mid \mathcal{U}^{k}=\left\{s \in \mathcal{S}(E) ; \operatorname{Im} j^{k}(s) \subset \mathcal{Q}^{k}\right\}$.

Definition 6.1.1. A differential equation $E_{1}$ at $j_{x}^{\alpha_{1}}\left(f^{1}\right) \in \tilde{J}^{\alpha_{1}}(N, Q)$ is said to be $k$-isomorphic to a differential equation $E_{2}$ at $j_{x}^{\alpha_{2}}\left(f^{2}\right) \in \tilde{J}^{\alpha_{2}}(N, Q)$, $k \geqslant 1$, if there are neighborhoods $U_{1}^{k}$ and $U_{2}^{k}$ of $j_{x}^{k}\left(f^{1}\right)$ and $j_{x}^{k}\left(f^{2}\right)$, respectively, and a diffeomorphism $\phi$ of $\vartheta_{1}=\beta^{k}\left(\vartheta_{1}^{k}\right)$ onto $\mho_{2}=\beta^{k}\left(ข_{2}^{k}\right)$ such that $\phi$ induces a one-to-one correspondence $\phi^{k}$ of $\mathcal{S}\left(E_{1}\right) \mid \mathcal{U}_{1}^{k}$ to $\delta\left(E_{2}\right) \mid \mathcal{U}_{2}^{k}$ by $\phi^{k}(s)=\phi \circ s$. Then $\phi$ is called a $k$-isomorphism of $E_{1}$ to $E_{2}$. An isomorphism is also called a 0 -isomorphism, and "0-isomorphic" means "isomorphic".

Proposition 6.1.1. If $E_{1}$ is $k$-isomorphic to $E_{2}$, then $E_{1}$ is l-isomorphic to $E_{2}$ for $l \geqslant k$.

Proof. Let $\phi$ be a $k$-isomorphism of $E_{1}$ to $E_{2}$ such that $\phi^{k}$ is a one-to-one map of $\delta\left(E_{1}\right) \mid U_{1}^{k}$ to $\delta\left(E_{2}\right) \mid \mathcal{U}_{2}^{k}$. We set, for $l \geqslant k$, $\mathcal{U}_{i}^{l}=\left(\rho_{k}^{l}\right)^{-1}\left(\mathcal{Q}_{i}^{k}\right)$. Then it is clear that $\phi$ induces a one-to-one map $\phi^{l}$ of $\mathcal{S}\left(E_{1}\right) \mid \mathcal{U}_{1}^{l}$ to $\mathcal{S}\left(E_{2}\right) \mid \mathcal{U}_{2}^{l}$. This proves Proposition 6.1.1.

Let $E_{i}$ and $E_{i}^{\prime}$ be differential equations at $j_{x}^{\alpha_{i}}\left(f^{i}\right) \in \tilde{J}^{\alpha_{i}}(N, Q)$ and $j_{x}^{\alpha_{i}^{\prime}}\left(f^{\prime i}\right) \in$ $J^{\alpha_{i}^{\prime}}\left(N, Q^{\prime}\right)$, respectively, for $i=1,2$, and let $\left(Q, Q^{\prime}, \pi\right)$ be a fibred manifold. We suppose $f^{i}=\pi \circ f^{i}$.

Lemma 6.1.1. Assume that $E_{i}$ is weakly $K$-reducible to $E_{i}^{\prime}$ by $\pi$, and that there is a $k$-isomorphism $\phi$ of $E_{1}$ to $E_{2}$. If there is a local diffeomorphism $\phi^{\prime}$ of $Q^{\prime}$ such that $\phi^{\prime} \circ \pi=\pi \circ \phi$, then $\phi^{\prime}$ is a $k^{\prime}$-isomorphism of $E_{1}^{\prime}$ to $E_{2}^{\prime}, k^{\prime}=$ $\max (k, K)$.

Proof. Since $E_{i_{\tilde{\tau}}}$ is weakly $K$-reducible to $E_{j}^{\prime}$, for any $\tilde{k} \geqslant K$ there exist neighborhoods $Q_{i}^{\tilde{k}}$ of $j_{x}^{\tilde{k}}\left(f^{i}\right)$ and $\mathcal{Q}_{i}^{\prime} \tilde{k}$ of $j_{x}^{k}\left(f^{\prime}\right)$, respectively, such that $\left(Q_{i}^{\tilde{k}}, \mathcal{Q}_{i}^{\prime \tilde{k}}, \pi^{\tilde{k}}\right)$ is a fibred manifold and such that $\pi$ induces a map of $\delta\left(E_{i}\right) \mid \mathcal{U}_{i}^{\tilde{k}}$ onto $\delta\left(E_{i}^{\prime}\right) \mid \mathcal{U}_{i}^{\prime \tilde{k}}$ for $i=1,2$. On the other hand, since $\phi$ is a $k$-isomorphism, by Proposition 6.1.1, $\phi$ is a $k^{\prime}$-isomorphsim, and $k^{\prime}=$ $\max (k, K)$. We may assume that $\phi^{k^{\prime}}$ is a one-to-one map of $\delta\left(E_{1}\right) \mid \mathcal{Q}_{1}^{k^{\prime}}$ to $\delta\left(E_{2}\right) \mid \mathcal{U}_{2}^{k^{\prime}}$. Let $s^{\prime} \in \mathcal{S}\left(E_{1}^{\prime}\right) \mid \mathcal{U}_{1}^{\prime k^{\prime}}$. Then we have an $s \in \mathcal{S}\left(E_{1}\right) \mid \mathcal{U}_{1}^{k^{\prime}}$ such that 
$s^{\prime}=\pi \circ s$. Thus we get $\phi^{\prime} \circ s^{\prime}=\phi^{\prime} \circ \pi \circ s=\pi \circ \phi \circ s$. Since $\phi \circ s \in$ $\delta\left(E_{2}\right) \mid \mathcal{U}_{2}^{k^{\prime}}$, we have $\pi \circ \phi \circ s \in \mathcal{S}\left(E_{2}^{\prime}\right) \mid \mathcal{Q}_{2}^{\prime k^{\prime}}$. This proves that $\phi^{\prime} \circ s^{\prime} \in$ $\mathcal{S}\left(E_{2}^{\prime}\right) \mid \mathcal{Q}_{2}^{\prime k^{\prime}}$. Therefore $\phi^{\prime}$ is a $k^{\prime}$-isomorphism of $E_{1}^{\prime}$ to $E_{2}^{\prime}$, and the proof is completed.

6.2. Let $\left(Q, Q^{\prime}, \pi\right)$ be a fibred manifold and let $\Gamma$ (resp. $\Gamma^{\prime}$ ) be a pseudo-group on $Q$ (resp. $Q^{\prime}$ ) which is complete at $(z, 1)$ for any $z \in Q$ (resp. at $\left(z^{\prime}, 1\right)$ for any $\left.z^{\prime} \in Q^{\prime}\right)$.

Let $E_{i}$ (resp. $E_{i}^{\prime}$ ) be a differential equation at ${ }_{x}^{\alpha_{i}}(f) \in \tilde{J}^{\alpha_{i}}(N, Q)$ (resp. $j_{x}^{\alpha_{i}^{\prime}}\left(f^{\prime}\right) \in \tilde{J}^{\alpha_{i}^{\prime}}\left(N, Q^{\prime}\right)$ ) such that $\mathbb{Q}\left(E_{i}\right)=\Gamma$ on a neighborhood of $f(x)$ (resp. $\mathcal{Q}\left(E_{i}^{\prime}\right)=\Gamma^{\prime}$ on a neighborhood of $f^{\prime}(x)$ with $\left.f^{\prime}=\pi \circ f\right), i=1,2$.

Let $\left\{\boldsymbol{\theta}_{j}^{\prime l}\right\}_{j=1}^{m_{i}^{\prime}}$ be a fundamental system of differential invariants of $\mathcal{E}_{\Gamma^{\prime}}$ at $j_{x}^{l}\left(f^{\prime}\right)$, and set $\theta^{\prime l}=\left(\theta_{1}^{\prime l}, \cdots, \theta_{m_{l}^{\prime}}^{\prime l}\right)$, where $l \geqslant$ the order of $\mathscr{E}_{T^{\prime}}$ at $\left(f^{\prime}(x), 1\right)$.

Let $j_{x}^{l}\left(g^{\prime}\right)$ be a point of $\tilde{J}^{l}\left(N, Q^{\prime}\right)$ near to $j_{x}^{l}\left(f^{\prime}\right)$ such that we can choose sufficiently small neighborhoods $\mathcal{V}^{\prime l}$ and $\mathcal{Q}^{\prime l}$ of $j_{x}^{l}\left(g^{\prime}\right)$ and $j_{x}^{l}\left(f^{\prime}\right)$, respectively, which satisfy $\mathscr{U}^{\prime \prime} \ni j_{x}^{l}\left(g^{\prime}\right)$ and $\mathcal{V}^{\prime l} \supset \mathcal{U}^{\prime \prime}$.

Theorem 6.2.1. Set $\theta^{\prime l}\left(j^{l}\left(f^{\prime}\right)\right)=\lambda^{\prime}$ and $\theta^{\prime l}\left(j^{l}\left(g^{\prime}\right)\right)=\mu^{\prime}$, and assume the following conditions:

(1) $\operatorname{dim} N \geqslant \operatorname{dim} Q$.

(2) $f \in \mathcal{S}\left(E_{1}\right)$.

(3) $\left(E_{i}, E_{i}^{\prime}\right)$ is a weakly $K$-reduced pair, and $E_{i}^{\prime}$ is l-regular at $x$.

(4) There is an m-isomorphism $\phi$ of $E_{1}$ to $E_{2}$ such that $g^{\prime}=\pi \circ \phi \circ f$.

(5) There is an element $\Delta \in D\left(\theta^{\prime \prime}, \Re\left(\Gamma^{\prime}\right)\right)$ such that $\Delta \circ \lambda^{\prime}=\mu^{\prime}$.

Then we have an $m^{\prime}$-isomorphism $\phi^{\prime}$ of $E_{1}^{\prime}$ to $E_{2}^{\prime}$ such that $\phi^{\prime} \circ \pi=\pi \circ \phi$ where $m^{\prime}=\max (m, K)$.

Proof. Since $\operatorname{dim} N \geqslant \operatorname{dim} Q^{\prime}$, by Lemma 5.1.2, $\mathcal{E}_{\Gamma^{\prime}}\left(l, x, f^{\prime}\right)$ and $\mathfrak{E}_{\Gamma^{\prime}}\left(l, x, g^{\prime}\right)$ which are defined on $\mathcal{U}^{\prime l}$ are $\Gamma^{\prime \prime}$-closed, where $\Gamma^{\prime \prime}=\Gamma^{\prime} \mid \mathcal{Q}^{\prime \prime}$. Since $\Delta \circ \lambda^{\prime}=\mu^{\prime}$, by Proposition 3.1.1 there is a 0-isomorphism $\varphi^{\prime}$ of $\mathscr{E}_{\Gamma^{\prime}}\left(l, x, f^{\prime}\right)$ to $\mathfrak{E}_{\Gamma^{\prime}}\left(l, x, g^{\prime}\right)$ such that $D\left(\theta^{\prime \prime}, \varphi^{\prime}\right)=\Delta$. Set $g^{\prime \prime}=\varphi^{\prime} \circ f^{\prime}$. Since $\mathfrak{L}_{\Gamma^{\prime}}\left(l, x, g^{\prime}\right)$ is $\Gamma^{\prime}$-automorphic, there exists an element $\gamma^{\prime} \in \Gamma^{\prime}$ such that $g^{\prime}=\gamma^{\prime} \circ g^{\prime \prime}$. Set $\phi^{\prime}=\gamma^{\prime} \circ \varphi^{\prime}$. Then we have $g^{\prime}=\phi^{\prime} \circ f^{\prime}$ and therefore $\pi \circ \phi \circ f=\phi^{\prime} \circ \pi \circ f$. Since $f$ is a submersion, we get $\pi \circ \phi=\phi^{\prime} \circ \pi$ on a neighborhood of $f(x)$. Therefore $\phi^{\prime}$ is an $m^{\prime}$-isomorphism of $E_{1}^{\prime}$ to $E_{2}^{\prime}$ by Lemma 6.1.1, and the proof is completed.

6.3. Example 3. Let $\mathcal{L}$ and $\Gamma$ be the same as in Example 1. Let $\phi \in \mathcal{R}(\Gamma)$, and $X=\xi\left(z_{1}\right) \partial / \partial z_{1}-\xi^{\prime}\left(z_{1}\right) z_{2} \partial / \partial z_{2}$ be a local cross section of $\mathcal{E}$. Then $\phi_{*} X$ is also a local cross section of $\mathcal{L}$, and therefore $\phi_{*} X$ is of the form $\eta\left(\tilde{z}_{1}\right) \partial / \partial \tilde{z}_{1}$ $-\eta^{\prime}\left(\tilde{Z}_{1}\right) \tilde{z}_{2} \partial / \partial \tilde{z}_{2}$, where $\tilde{z}_{i}=z_{i} \circ \phi=\phi^{i}(i=1,2)$. Thus we have

$$
\frac{D\left(\phi^{1}, \phi^{2}\right)}{D\left(x_{1}, x_{2}\right)}\left(\begin{array}{c}
\xi\left(z_{1}\right) \\
-\xi^{\prime}\left(z_{1}\right) z_{2}
\end{array}\right)=\left(\begin{array}{c}
\eta\left(\tilde{z}_{1}\right) \\
-\eta^{\prime}\left(\tilde{z}_{1}\right) \tilde{z}_{2}
\end{array}\right) \text {, }
$$


so that

$$
\begin{gathered}
\eta\left(\tilde{z}_{1}\right)=\eta\left(\phi^{1}\left(z_{1}, z_{2}\right)\right)=\frac{\partial \phi^{1}}{\partial z_{1}} \cdot \xi\left(z_{1}\right)-\frac{\partial \phi^{1}}{\partial z_{2}} \cdot \xi^{\prime}\left(z_{1}\right) \cdot z_{2} \\
-\eta^{\prime}\left(\tilde{z}_{1}\right) \tilde{z}_{2}=-\eta^{\prime}\left(\phi^{1}\left(z_{1}, z_{2}\right)\right) \phi^{2}=\frac{\partial \phi^{2}}{\partial z_{1}} \cdot \xi\left(z_{1}\right)-\frac{\partial \phi^{2}}{\partial z_{2}} \cdot \xi^{\prime}\left(z_{1}\right) z_{2}
\end{gathered}
$$

On the other hand,

$$
\begin{aligned}
&-\eta^{\prime}\left(\tilde{z}_{1}\right) \tilde{z}_{2}=-\left(\frac{\partial \eta\left(\phi^{1}\left(z_{1}, z_{2}\right)\right)}{\partial z_{1}} \cdot \frac{\partial z_{1}}{\partial \tilde{z}_{1}}+\frac{\partial \eta\left(\phi^{1}\left(z_{1}, z_{2}\right)\right)}{\partial z_{2}} \cdot \frac{\partial z_{2}}{\partial \tilde{z}_{1}}\right) \phi^{2} \\
&=-\left\{\left[\frac{\partial^{2} \phi^{1}}{\partial z_{1}^{2}} \cdot \xi\left(z_{1}\right)+\frac{\partial \phi^{1}}{\partial z_{1}} \cdot \xi^{\prime}\left(z_{1}\right)-\frac{\partial^{2} \phi^{1}}{\partial z_{1} \partial z_{2}} \cdot \xi^{\prime}\left(z_{1}\right) \cdot z_{2}\right.\right. \\
&\left.-\frac{\partial \phi^{1}}{\partial z_{2}} \cdot \xi^{\prime \prime}\left(z_{1}\right) \cdot z_{2}\right] \frac{\partial z_{1}}{\partial \tilde{z}_{1}}+\left[\frac{\partial^{2} \phi^{1}}{\partial z_{1} \partial z_{2}} \cdot \xi\left(z_{1}\right)\right. \\
&\left.\left.-\frac{\partial^{2} \phi^{1}}{\partial z_{2}^{2}} \cdot \xi^{\prime}\left(z_{1}\right) \cdot z_{2}-\frac{\partial \phi^{1}}{\partial z_{2}} \cdot \xi^{\prime}\left(z_{1}\right)\right] \frac{\partial z_{2}}{\partial \tilde{z}_{1}}\right\} \cdot \phi^{2} .
\end{aligned}
$$

Since $\xi$ is arbitrary, we get the following equations:

$$
\begin{gathered}
\frac{\partial \phi^{2}}{\partial z_{1}}+\left(\frac{\partial^{2} \phi^{1}}{\partial z_{1}^{2}} \cdot \frac{\partial z_{1}}{\partial \tilde{z}_{1}}+\frac{\partial^{2} \phi^{1}}{\partial z_{1} \partial z_{2}} \cdot \frac{\partial z_{2}}{\partial \tilde{z}_{1}}\right) \cdot \phi^{2}=0 \\
\frac{\partial \phi^{2}}{\partial z_{2}} \cdot z_{2}-\left\{\left(\frac{\partial \phi^{1}}{\partial z_{1}}-\frac{\partial^{2} \phi^{1}}{\partial z_{1} \partial z_{2}} \cdot z_{2}\right) \cdot \frac{\partial z_{1}}{\partial \tilde{z}_{1}}+\left(\frac{\partial^{2} \phi^{1}}{\partial z_{2}^{2}} \cdot z_{2}+\frac{\partial \phi^{1}}{\partial z_{2}}\right) \cdot \frac{\partial z_{2}}{\partial \tilde{z}_{1}}\right\} \cdot \phi^{2}=0, \\
\frac{\partial \phi^{1}}{\partial z_{2}}=0 .
\end{gathered}
$$

Therefore $\phi^{1}$ is a function of the form $\alpha\left(z_{1}\right)$, and from the first and the second of the above equations we get

$$
\frac{\partial \phi^{2}}{\partial z_{1}}+\frac{\partial^{2} \phi^{1}}{\partial z_{1}^{2}} \cdot \frac{\partial z_{1}}{\partial \tilde{z}_{1}} \cdot \phi^{2}=0, \quad \frac{\partial \phi^{2}}{\partial z_{2}} \cdot z_{2}-\frac{\partial \phi^{1}}{\partial z_{1}} \cdot \frac{\partial z_{1}}{\partial \tilde{z}_{1}} \cdot \phi^{2}=0
$$

which are equivalent to

$$
\frac{\partial \phi^{1}}{\partial z_{1}} \cdot \frac{\partial \phi^{2}}{\partial z_{1}}+\frac{\partial^{2} \phi^{1}}{\partial z_{1}^{2}} \cdot \phi^{2}=0, \quad \frac{\partial \phi^{2}}{\partial z_{2}} \cdot z_{2}-\phi^{2}=0,
$$

since $\partial z_{1} / \partial \tilde{z}_{1}=\left(\partial \phi^{1} / \partial z_{1}\right)^{-1}$. By the above second equation we see that $\phi^{2}\left(z_{1}, z_{2}\right)$ is of the form $\beta\left(z_{1}\right) \cdot z_{2}$. Replacing $\phi^{1}\left(z_{1}\right)$ and $\phi^{2}\left(z_{1}, z_{2}\right)$ by $\alpha\left(z_{1}\right)$ and $\beta\left(z_{1}\right) \cdot z_{2}$ respectively, from the above first equation we get the following 
ordinary differential equation with respect to one unknown function $\beta$ :

$$
\beta^{\prime}\left(z_{1}\right)=-\frac{\alpha^{\prime \prime}\left(z_{1}\right)}{\alpha^{\prime}\left(z_{1}\right)} \cdot \beta\left(z_{1}\right),
$$

the general solution of which is $c / \alpha^{\prime}\left(z_{1}\right)$. This proves that if $\phi \in \mathcal{U}(\Gamma)$ then $\phi$ must be of the form $\phi^{1}\left(z_{1}, z_{2}\right)=\alpha\left(z_{1}\right)$ and $\phi^{2}\left(z_{1}, z_{2}\right)=c \cdot z_{2} / \alpha^{\prime}\left(z_{1}\right)$.

Conversely, if a local diffeomorphism $\phi$ of $\mathbf{R}_{*}^{2}$ is of the form

$$
\phi^{1}\left(z_{1}, z_{2}\right)=\alpha\left(z_{1}\right), \quad \phi^{2}\left(z_{1}, z_{2}\right)=\frac{c \cdot z_{2}}{\alpha^{\prime}\left(z_{1}\right)},
$$

it is easy to see that $\phi^{(1) *}\left(z_{2} \cdot \partial z_{1} / \partial x_{i}\right)=c \cdot\left(z_{2} \cdot \partial z_{1} / \partial x_{i}\right), i=1,2$. Therefore we get $\phi \in \Re(\Gamma)$.

Now we can see such an example that the compatibility of reduction and equivalence holds. Let $E_{i}$ be the differential equation at $j_{x_{0}}^{1}\left(f_{i}\right) \in \tilde{J}^{1}\left(\mathbf{R}^{2}, \mathbf{R}_{*}^{2}\right)$ generated by

$$
z_{2} \cdot \frac{\partial z_{1}}{\partial x_{1}}-\alpha_{i}\left(x_{1}, x_{2}\right), \quad z_{2} \cdot \frac{\partial z_{1}}{\partial x_{2}}-\beta_{i}\left(x_{1}, x_{2}\right)
$$

where

$$
\alpha_{i}(x)=\left[z_{2} \cdot \frac{\partial z_{1}}{\partial x_{1}}\right]\left(j_{x}^{1}\left(f_{i}\right)\right), \quad \beta_{i}(x)=\left[z_{2} \cdot \frac{\partial z_{1}}{\partial x_{2}}\right]\left(j_{x}^{1}\left(f_{i}\right)\right) .
$$

Assume that $\alpha_{i}(x) \neq 0$, and denote by $E_{i}^{\prime}$ the differential equation at $j_{x_{0}}^{1}\left(f_{i}^{\prime}\right) \in$ $\tilde{J}^{1}\left(\mathbf{R}^{2}, \mathbf{R}\right)$ generated by

$$
\frac{\partial z_{1}}{\partial x_{2}} / \frac{\partial z_{1}}{\partial x_{1}}-\beta_{i} / \alpha_{i}
$$

where $f_{i}^{\prime}=\pi \circ f_{i}$ and $\pi$ is the projection of $\mathbf{R}_{*}^{2}$ onto $\mathbf{R}$ defined by $z_{1}=$ $\pi\left(z_{1}, z_{2}\right)$.

Suppose $E_{1}$ is 0 -isomorphic to $E_{2}$, and $\phi$ is such a 0 -isomorphism. Since $\mathcal{Q}\left(E_{i}\right)=\Gamma$ on a neighborhood of $f_{i}\left(x_{0}\right)$, we get $\phi \in \mathcal{N}(\Gamma)$. By the above calculation of $\Re(\Gamma)$, we have $\phi^{(1) *}\left(z_{2} \cdot \partial z_{1} / \partial x_{j}\right)=a \cdot\left(z_{2} \cdot \partial z_{1} / \partial x_{j}\right)$, where $a$ is constant $\neq 0$. Therefore, if $E_{1}$ if 0 -isomorphic to $E_{2}$, there is a constant $b \neq 0$ such that $\alpha_{2}=b \cdot \alpha_{1}$ and $\beta_{2}=b \cdot \beta_{1}$. Consequently we get $\beta_{2} / \alpha_{2}=$ $\beta_{1} / \alpha_{1}$ and therefore $E_{1}^{\prime}=E_{2}^{\prime}$, in particular, $E_{1}^{\prime}$ is 0-isomorphic to $E_{2}^{\prime}$.

\section{Closedness of the pseudo-group of isometries}

7.1. Let $\Theta^{\alpha}$ be the sheaf of germs of local Killing vector fields of $\mathbf{R}^{q}$, and let $\Gamma\left(\Theta^{q}\right)$ be the pseudo-group of local isometries of $\mathbf{R}^{q}$. Denote by $\left\{x_{1}, \cdots, x_{n}\right\}$ (resp. $\left\{z_{1}, \cdots, z_{q}\right\}$ ) the coordinate system on $\mathbf{R}^{n}$ (resp. $\mathbf{R}^{q}$ ), and set $\tilde{\rho}_{j}=\sum_{i=1}^{q}\left(\partial^{2} z_{i} / \partial x_{j}^{2}\right)^{2}$. Then $\tilde{\rho}_{j}$ is a differential invariant of $\Theta^{q}$ at any 
$j_{x}^{2}(f) \in \tilde{J}^{2}\left(\mathbf{R}^{n}, \mathbf{R}^{q}\right)$. In the following lemma, as for the property $P(2, x, f)$, refer to $\$ 8$.

Lemma 7.1.1. $\Gamma\left(\Theta^{q}\right)$ satisfies the property $P(2, x, f)$.

Proof. Let $\phi$ be a local diffeomorphism of $\mathbf{R}^{q}$ such that $\phi^{(2) *} \tilde{\rho}_{2}=\tilde{\rho}_{1}$. Then we get $\partial^{2} \phi_{i} / \partial z_{j} \partial z_{k}=0$ and $\sum_{h=1}^{q} \partial \phi_{i} / \partial z_{h} \cdot \partial \phi_{j} / \partial z_{h}=\delta_{i j}(1<i, j, k<q)$, where $\phi_{i}=z_{i} \circ \phi$. This implies that $\phi_{i}(z)=\sum_{j=1}^{q} a_{i j} z_{j}+b_{i},\left(a_{i j}\right) \in O(q)$, on the domain of $\phi$, so that $\phi \in \Gamma\left(\Theta^{q}\right)$, that is, $\Gamma\left(\Theta^{q}\right)=\left\{\phi\right.$; local diffeo. of $\mathbf{R}^{q}$, $\left.\phi^{(2) *} \tilde{\rho}_{1}=\tilde{\rho}_{1}\right\}$. Thus $\Theta^{q}$ satisfies the property $P(2, x, f)$ for any $(x, f)$.

Set $\lambda_{j}(x)=\tilde{\rho}_{j}\left(j_{x}^{2}(f)\right), 1 \leqslant j \leqslant n$.

Proposition 7.1.1. If $\lambda_{j}$ is a submersion on a neighborhood of $x_{0}$ for some $j$, then $\Gamma\left(\Theta^{q}\right)$ is 2-closed at $\left(x_{0}, f\right)$.

Proof. We shall show that $\Gamma\left(\Theta^{q}\right)$ is 2-closed at $\left(x_{0}, f\right)$ if $\lambda_{1}$ is a submersion. Let $V^{2}$ be a neighborhood of $j_{x_{0}}^{2}(f)$, and set

$$
S\left(V^{2}\right)=\bigcup_{s \in \delta\left(\Theta^{q}\left(l, x_{02} f\right)\right)} \operatorname{graph}\left(j^{2}(s)\right) \cap V^{2} .
$$

If $\phi \in \mathbb{Q}\left(\theta^{q}\left(l, x_{0}, f\right)\right)$, we have $\phi^{(2) * \tilde{\rho}_{1}}=\tilde{\rho}_{1}$ on $S\left(V^{2}\right)$, because $\phi^{(2)} \mid S\left(V^{2}\right)$ is a local transformation of $S\left(V^{2}\right)$ and $\tilde{\rho}_{1}\left(j_{x}^{2}(s)\right)=$ $\tilde{\rho}_{1}\left(j_{x}^{2}(t)\right)=\lambda_{1}(x)$ for any two $s$ and $t \in \delta\left(\Theta^{q}\left(l, x_{0}, f\right)\right)$. Let $\left\{x_{1}, \cdots, x_{n}, z_{1}, \cdots, z_{q}, \cdots, p_{j}^{i}, \cdots, p_{j k}^{i}, \cdots\right\}$ be the natural coordinate system on $\tilde{J}^{2}\left(\mathbf{R}^{n}, \mathbf{R}^{q}\right)$. Then we may assume that $V^{2}$ possesses a product structure $\mathscr{H} \times \mathscr{Z} \times \mathscr{P}$, where $\mathcal{H}, \mathscr{Z}$ or $\mathscr{P}$ is a cubic neighborhood of a point of $\mathbf{R}^{n}, \mathbf{R}^{q}$ or $\mathbf{R}^{m}$ such that $\left\{x_{1}, \cdots, x_{n}\right\},\left\{z_{1}, \cdots, z_{1}\right\}$ or $\left\{p_{1}^{1}, \cdots, p_{j}^{i}, \cdots, p_{j k}^{i}, \cdots\right\}$ is the coordinate system on $\mathcal{H}, \mathcal{Z}$ or $\mathscr{P}$. Moreover we may assume that $\mathscr{P}$ possesses a product structure $\mathscr{P}^{1} \times \mathscr{P}^{2}$, where $\mathscr{P}^{1}$ is a cubic neighborhood of a point of $\mathbf{R}^{q}$ such that $\left\{p_{11}^{1}, \cdots, p_{11}^{q}\right\}$ is the coordinate system on $\mathcal{P}^{1}$ and $\mathcal{P}^{2}$ is a cubic neighborhood of a point of $\mathbf{R}^{m-q}$ such that $\left\{\cdots, p_{j}^{i}, \cdots, p_{h k}^{i}, \cdots\right\}(h \neq 1$ or $k \neq 1)$ is the coordinate system on $\mathscr{P}^{2}$. Let $\pi$ be the natural projection of $V^{2} \rightarrow \mathscr{P}^{1}$. We shall prove that $\pi\left(S\left(V^{2}\right)\right)$ is open in $\mathscr{P}^{1}$. Let $\phi \in \Gamma\left(\Theta^{q}\right)$. Then we have $\phi_{i}(z)=$ $\sum_{j=1}^{q} a_{i j}(\phi) z_{j}+b_{i}, \quad\left(a_{i j}(\phi)\right) \in O(q)$ on the domain of $\phi$. For $s \in$ $\mathcal{S}\left(\Theta^{q}\left(l, x_{0}, f\right)\right)$, we set $s^{\prime}=\phi \circ s$. Then

$$
\frac{\partial^{2} s_{i}^{\prime}}{\partial x_{1}^{2}}=\sum_{j=1}^{q} a_{i j}(\phi) \cdot \frac{\partial^{2} s_{j}}{\partial x_{1}^{2}} .
$$

Since $\lambda_{1}$ is a submersion on a neighborhood $थ$ of $x_{0}, \lambda_{1}(W)$ is open in $\mathbf{R}$. Since

$$
\tilde{\rho}_{1}\left(j_{x}^{2}(s)\right)=\sum_{i=1}^{q}\left(\frac{\partial^{2} s_{i}}{\partial x_{1}^{2}}\right)^{2}=\lambda_{1}(x), \quad \frac{\partial^{2} s}{\partial x_{1}^{2}}(x)=\left(\frac{\partial^{2} s_{1}}{\partial x_{1}^{2}}(x), \cdots, \frac{\partial^{2} s_{q}}{\partial x_{1}^{2}}(x)\right)
$$


is a point of $(q-1)$-sphere $S^{q-1}\left(\lambda_{1}(x)\right)$ in $\mathbf{R}^{q}$ with the radius $\sqrt{\lambda_{1}(x)}$. On the other hand, $O(q)$ acts transitively on $S^{q-1}\left(\lambda_{1}(x)\right)$. Since $\Gamma\left(\theta^{q}\right) \ni \phi \rightarrow\left(a_{i j}(\phi)\right)$ $\in O(q)$ is onto and since $\theta^{q}\left(l, x_{0}, f\right)$ admits $\Gamma\left(\Theta^{q}\right),\left\{\left(\partial^{2}(\phi \circ s) / \partial x_{1}^{2}\right)(x)\right.$; $\left.\phi \in \Gamma\left(\Theta^{q}\right), \quad x \in \mathcal{W}\right\}$ is open in $\mathbf{R}^{q}$. Therefore $\pi\left(S\left(V^{2}\right)\right)=$ $\left\{\left(\partial^{2}(\phi \circ s) / \partial x_{1}^{2}\right)(x) ; \phi \in \Gamma\left(\Theta^{q}\right), x \in \mathcal{Q}\right\} \cap \mathscr{P}^{1}$ is open in $\mathscr{P}^{1}$. Now $\tilde{\rho}_{1}$ is considered as a function on $\mathcal{P}^{1}$. Therefore the equality $\phi^{(2) *} \tilde{\rho}_{1}=\tilde{\rho}_{1}$ on $S\left(V^{2}\right)$ means the equality $\phi^{(2) *} \tilde{\rho}_{1}=\tilde{\rho}_{1}$ on $\pi\left(S\left(V^{2}\right)\right)$. Since $\pi\left(S\left(V^{2}\right)\right)$ is open in $\mathcal{P}^{1}$, we get $\phi^{(2) *} \tilde{\rho}_{1}=\tilde{\rho}_{1}$ as a function. As was proved in Lemma 7.1.1, the equation $\phi^{(2) *} \tilde{\rho}_{1}=\tilde{\rho}_{1}$ implies $\phi \in \Gamma\left(\Theta^{q}\right)$. Therefore we have proved that if $\phi \in \mathbb{Q}\left(\Theta^{q}\left(l, x_{0}, f\right)\right)$, then $\phi \in \Gamma\left(\Theta^{q}\right)$. This implies that $\Gamma\left(\Theta^{q}\right)$ is $l$-closed at $\left(x_{0}, f\right)$ for $l \geqslant 2$, and hence the proof is completed.

\section{Appendix 1 (Completeness of pseudo-groups)}

8.1. Let $\Gamma$ be a pseudo-group on $Q$ such that $\varrho_{\Gamma}$ is an $N$-regular weak Lie algebra sheaf, and let $\left\{\theta_{j}^{l}\right\}_{j=1}^{m_{1}}$ be a fundamental system of differential invariants of $\mathfrak{E}_{T}$ at $j_{x}^{l}(f) \in \tilde{J}^{l}(N, Q)$.

Definition 8.1.1. $\Gamma$ is said to satisfy the property $P(l, x, f)$ (resp. $P(\infty, x, f))$ if the following statement holds: Let $V^{l}$ be a sufficiently small neighborhood of $j_{x}^{l}(f)$ on which $\theta_{j}^{l}\left(1 \leqslant j \leqslant m_{l}\right)$ is defined, and let $\phi$ be a local diffeomorphism of $Q$ such that $\phi^{(l)}$ maps an open subset $W^{l}\left(\exists j_{x^{\prime}}^{l}\left(f^{\prime}\right)\right)$ of $V^{l}$ into $V^{l}$. Then ' $\phi$, a restriction of $\phi$ to a neighborhood ' $W \subset \beta^{l}\left(W^{l}\right)$ of $f^{\prime}\left(x^{\prime}\right)$, is in $\Gamma$ if and only if $\phi^{(l) *} \theta_{j}^{l}=\theta_{j}^{l}\left(1 \leqslant j \leqslant m_{l}\right)$ on a neighborhood ${ }^{\prime} W^{l}=\left(\beta^{l}\right)^{-1}\left({ }^{\prime} W\right) \cap W^{l}\left(\exists j_{x^{\prime}}^{l}\left(f^{\prime}\right)\right)$ for an integer $l \geqslant 0$ (resp. for any integer $l \geqslant 0)$.

Let $\Gamma$ be a pseudo-group on $Q$, and let $f$ be a diffeomorphism of a neighborhood of $x \in Q$ to a neighborhood of $f(x) \in Q$.

Definition 8.1.2. $\Gamma$ is said to be complete at $(x, f)$ if the following conditions are satisfied:

(1) $\mathscr{L}_{\Gamma}$ is a regular Lie algebra sheaf around $f(x)$.

(2) $\Gamma$ satisfies the property $P(\infty, x, f)$.

Proposition 8.1.1. Suppose a pseudo-group $\Gamma$ on $Q$ satisfies the following conditions:

(i) $\mathfrak{L}_{T}$ is a regular weak Lie algebra sheaf.

(ii) $\Gamma$ satisfies the property $P(\infty, x, f)$.

Then $\Gamma$ is complete at $(x, f)$.

Proof. Let $X$ be a vector field on a neighborhood $U \subset Q$ of $f(x)$, and assume that $\mathbf{F}_{z}(X(z)) \in L(z)$ for any $z \in U$. As for the definition of $F_{z}$, refer to [6, p. 462]. Let $\varphi_{t}$ be the local 1-parameter group of local transformation of $U$ generated by $X$. Since the condition that $F_{z}(X(z)) \in L(z)$ for any $z \in U$ implies that $X^{(l)}$ is a local cross section of $D_{\Gamma}^{(l)}$ defined on a neighborhood of 
$j_{x}^{l}(f)$ for any $l \geqslant 0$. Since $\Gamma$ satisfies the property $P(\infty, x, f)$, we can easily see that $\varphi_{t} \in \Gamma$. Therefore $X$ is a local cross section of $\varrho_{\Gamma}$. This means that $\varrho_{\Gamma}$ is a Lie algebra sheaf. Therefore $\Gamma$ is complete at $(x, f)$, and the proof is completed.

Now let $\Gamma$ be a pseudo-group on $Q$ such that $\mathcal{L}_{\Gamma}$ is a regular weak Lie algebra sheaf.

Proposition 8.1.2. $\Gamma$ is complete at $\left(z_{0}, 1\right)$ if and only if $\Gamma$ is locally defined at $z_{0}$ by a system of differential equations $(A)^{l}$ at $j_{z_{0}}^{l}(1) \in \tilde{J}^{l}(Q, Q)$ for an integer $l$.

The proof is given in [6, Propositions 8.1, 8.2].

\section{Appendix 2 (Order of pseudo-groups)}

9.1. Let $\mathcal{L}$ be an $N$-regular weak Lie algebra sheaf on $Q$.

Proposition 9.1.1. There is an integer $K$ such that, for any $k \geqslant K, \mathcal{L}(k+$ $1, x, f)=p \mathcal{L}(k, x, f)$ on a neighborhood of $j_{x}^{k+1}(f) \in \tilde{J}^{k+1}(N, Q)$, where $p \mathfrak{L}(k, x, f)$ is the standard prolongation of $\mathcal{L}(k, x, f)$.

The proof is given in [6, Lemma 4.1].

Definition 9.1.1. We denote by $K_{0}$ the minimum integer $K$ satisfying Proposition 9.1.1. The integer $K_{0}$ is called the order of $\mathcal{L}$ at $(x, f)$.

Let $\Gamma$ be a pseudo-group on $Q$, and let $E$ be a differential equation at $j_{x}^{\alpha}(f) \in \tilde{J}^{\alpha}(N, Q)$ where $f$ is a solution of $E$.

Definition 9.1.2. $E$ is said to be $\Gamma$-automorphic if the following conditions are satisfied:

(1) For any $\phi \in \Gamma, \phi \circ f$ is a solution of $E$ if the composite is defined.

(2) Any solution $s$ of $E$ near to $f$ is of the form $\phi \circ f$ for some $\phi \in \Gamma$.

\section{References}

[1] H. H. Johnson, Classical differential invariants and applications to partial differential equations, Math. Ann. 148 (1962) 308-329.

[2] M. Kuranishi, Lectures on exterior differential systems, Tata Inst. Fundamental Research, Bombay, 1962.

[3] S. Lie, Verwertung des Gruppenbegriffes für Differentialgleichungen. I, Leipzig Berichte (1895) 261-322.

[4] S. Lie, Zur allgemeinen Theorie der partiellen Differentialgleichungen beliebiger Ordunung, Leipzig Berichte (1895) 53-128.

[5] I. M. Singer \& S. Sternberg, The infinite group of Lie and Cartan, J. Analyse. Math. 15 (1965) 1-114.

[6] K. Ueno, Existence and equivalence theorems of automorphic systems, Publ. Res. Inst. Math. Sci. Kyoto Univ. 11 (1976) 461-482.

[7] E. Vessiot, Sur l'intégration des systèmes differentiels qui admettent des groupes continus de transformation, Acta Math. 28 (1904) 307-349. 
